1 Short Title: Exploring functional diversity of tobacco aquaporins.

2 Author for contact: Annamaria De Rosa ( annamaria.derosa@anu.edu.au )

\title{
Exploring functional diversity of Nicotiana tabacum Aquaporins
}

\author{
Annamaria De Rosa ${ }^{*}$, John R Evans ${ }^{1}$ and Michael Groszmann ${ }^{1}$ \\ ${ }^{1}$ ARC Centre of Excellence for Translational Photosynthesis, Research School of Biology, Australian National \\ University, Canberra, ACT 2601, Australia \\ * For correspondence (email annamaria.derosa@anu.edu.au)
}

\section{Sentence Summary:}

Diverse tobacco PIP, TIP and NIP aquaporin isoforms were functionally characterised using highthroughput yeast-based assays, assessing their transport capabilities for key plant solutes: water, $\mathrm{H}_{2} \mathrm{O}_{2}$, boric acid and urea.

\section{Author Contributions:}

MG and JRE conceived original research plans; ADR performed yeast screening experiments and subcellular localisation analyses; ADR and MG performed 3D protein homology modeling analyses; $A D R$, JRE and MG analyzed the data and wrote the manuscript. ADR agrees to serve as the author responsible for contact and ensures communication.

\section{Funding Information:}

This work was funded by the Australian Government through the Australian Research Council Centre of Excellence for Translational Photosynthesis (CE140100015).

\section{Abstract}

Aquaporins (AQPs) are involved in a variety of vital plant physiological processes, including water relations, development, stress responses and photosynthesis. Nicotiana tabacum (tobacco) has 84 $A Q P$ genes due to its recent hybridisation and allotetraploid genome. We functionally characterised a diverse subset of tobacco AQPs spanning the 3 largest AQP subfamilies, selecting nine isoforms from the PIPs, TIPs, and NIPs. Using high-throughput yeast-based functional assays, we determined AQP permeability to water, hydrogen peroxide $\left(\mathrm{H}_{2} \mathrm{O}_{2}\right)$, boric acid $(B A)$ and urea. For each $A Q P$, subcellular localisations in planta were established using GFP translational fusions. From 3D protein homology modelling, we found that the monomeric pore shape/size, selectivity filter region and NPA motifs is insufficient to comprehensively predict their transport capabilities. PIPs had the narrowest pore diameter and were permeable to water, $\mathrm{H}_{2} \mathrm{O}_{2}$ and BA. The pore in TIPs was wider and more cylindrical in shape than for the PIPs. TIP1;1s was permeable to all four substrates tested, and is highly expressed in leaves and flowers, suggesting it functions in multiple roles. By contrast, NIP5;1t, with a larger pore size than the NtTIPs, is only expressed in young flowers and enhanced permeability only to BA. Its homolog in Arabidopsis (AtNIP5;1) has the same substrate specificity and functions as a boron channel. 


\section{Introduction}

Aquaporins (AQPs) constitute a major family of integral membrane channel proteins found across all kingdoms of life (Abascal et al. 2014), becoming most diversified in number and subfamilies in plants (Groszmann et al. 2017; Abascal et al. 2014). Plant AQPs play a vital role in diverse physiological processes, including water relations, growth and development, stress responses, and photosynthesis (Hachez et al. 2006; Groszmann et al. 2017; Chaumont and Tyerman 2017). This range in cellular functions reflects a capability for transporting a wide variety of substrates including water, nitrogen compounds (e.g. ammonia, urea and nitrate), gases (e.g. carbon dioxide, oxygen), hydrogen peroxide, metalloids (e.g. boron, silicon), and ions (Gomes et al. 2009; Pommerrenig et al. 2015; Hove and Bhave 2011; Choi and Roberts 2007; Zwiazek et al. 2017; Byrt et al. 2017; Bienert et al. 2013; Liu et al. 2020). However, functional characterisation of AQPs is quite limited. Elucidating substrate transport profiles of AQPs is a key component for understanding their potential roles in plants and for enabling their use in crop improvement.

Aquaporins assemble as tetrameric complexes, with each monomer forming a functional pore created by six membrane spanning helices, five connecting loops and two shorter helices. At the centre of the AQP tetramer there is a fifth central and functioning pore (Pommerrenig et al. 2015; Kirscht et al. 2016; Törnroth-Horsefield et al. 2006). Although the gross tertiary structure of AQP is highly conserved across organisms, slight deviations in structural and functional characteristics between isoforms contribute to differences in their transport selectivity. Such characteristics include pore dimensions (pore diameter and overall morphology), chemical properties and flexibilities of pore-lining residues, and specific configurations of residues at key constriction points (Luang and Hrmova 2017). Higher plant AQPs divide into five phylogenetically distinct sub-families, namely the Plasma membrane Intrinsic Proteins (PIPs), Tonoplast Intrinsic Proteins (TIPs), Small basic Intrinsic Proteins (SIPs), Nodulin 26-like Intrinsic Proteins (NIPs), and X Intrinsic Proteins (XIPs) (Danielson and Johanson 2008; Johanson and Gustavsson 2002; Kaldenhoff and Fischer 2006). Within each of these sub-families, there can be diversity in permeating substrate selectivity and specific organelle membrane integration (Maurel et al. 2008). Subfamily-specific substrate specificities in plant AQPs have been attributed to diversity in the aromatic arginine (ar/R) selectivity filter (SF) which forms the first constriction site towards the extracellular side of the pore (Hove and Bhave 2011). Variation in this site largely determines the substrates able to permeate across the membrane through the AQP (Hove and Bhave 2011; Sui et al. 2001). Dual Asn-Pro-Ala (NPA) motifs located at the centre of the pore act as a second constriction, with variation in residue composition contributing to selectivity for substrates such as ammonia, boric acid (BA) and urea (Wu and Beitz 2007; Hove and Bhave 2011).

Nicotiana tabacum (tobacco) arose from a recent hybridization and its allotetraploid genome contains 84 AQP genes (De Rosa et al. 2020; Ahmed et al. 2020; Groszmann et al. 2021). The multiplicity of $A Q P$ gene isoforms and relatedness to horticulturally important crops such as tomato, potato, eggplant and capsicum, makes tobacco a favourable study species for translating key findings into crops (De Rosa et al. 2020).

Teasing apart the complexities of aquaporin biology in planta can be difficult. Plants have many AQP isoforms, with some having redundancy of function under certain environmental conditions (Abascal et al. 2014; Fox et al. 2017). As such, modification of aquaporin expression within the plant, via over expression or down regulation of a specific AQP, may also affect the expression of closely related isoforms (Bi et al. 2015; Kaldenhoff et al. 2007). Functional characterisation of plant AQPs is often accomplished through heterologous expression systems, such as yeast (Kaldenhoff et al. 2007), allowing for assignment of substrate specificity to an AQP homo-tetramer in isolation from other isoforms and other confounding external regulatory mechanisms. We developed a high-throughput micro-cultivation-based yeast assay to study AQP function and used it to develop substrate profiles 
for the entire Arabidopsis PIP family (Groszmann et al. in preparation). Here we extend the application of this platform to screen a diverse subset of tobacco AQPs spanning the 3 largest AQP subfamilies (PIPs, TIPs and NIPs). These AQPs isoforms were chosen based on homology to already characterised $A Q P s$ in other species or on gene expression characteristics within the plant which might implicate diverse functional roles. Three PIP1 genes were chosen: NtPIP1;5s (NtAQP1), NtPIP1;1t and NtPIP1;3t, each sharing more than $90 \%$ homology in gene sequence. NtPIP1;5s (NtAQP1) is an established $\mathrm{CO}_{2}$ permeable AQP isoform, that enhanced $\mathrm{CO}_{2}$ diffusion and photosynthetic efficiency in planta (Uehlein et al. 2003; Flexas et al. 2006). Here we assessed permeability to water, $\mathrm{H}_{2} \mathrm{O}_{2}$, $\mathrm{BA}$ and urea, but not $\mathrm{CO}_{2}$. Two PIP2 genes, NtPIP2;4s and NtPIP2;5t, were chosen as representative isoforms from distinct phylogenetic sub-clades within the PIP2 phylogeny. From the TIPs, NtTIP1;1s was chosen as a gene highly expressed throughout the plant (De Rosa et al. 2020) and potentially permeable to range of solutes, while NtTIP2;5t, a TIP predominantly expressed in roots (De Rosa et al. 2020), had high homology to AtTIP2;1, an established transporter of nitrogen compounds (urea and ammonium) (Loqué et al. 2005; Liu et al. 2003). Representative isoforms from two distinct subclasses within the NIP subfamily were selected. NtNIP5;1s belongs to the NIP II class and is homologous to the boronpermeable AtNIP5;1 (Takano et al. 2006; Pommerrenig et al. 2015). NtNIP2;1s belongs to a distinct class of NIPs (NIP III), which transports a diverse range of metalloid compounds including the important micronutrients boron and silicon.

We used our high-throughput yeast-based functional system to test for water, hydrogen peroxide $\left(\mathrm{H}_{2} \mathrm{O}_{2}\right), \mathrm{BA}$ and urea permeability. Subcellular localisation of each AQP was visualised in planta with an Arabidopsis expression system. Substrate permeability and sub-cellular localisation data were collated with gene expression data and 3D homology modelling to characterise these tobacco AQPs.

\section{Results}

\section{Tobacco Aquaporin localisation in Yeast}

Nine diverse NtAQP isoforms, from the PIP, NIP and TIP sub-families (Figure 1) were functionally characterised. N-terminal GFP-AQP fusions were used to establish whether NtAQP proteins integrated into the plasma membrane (PM) of yeast (Figure $2 A-I)$, a necessary condition for attributing AQPfacilitated diffusion into the cell. Optically thin focal plane images of each yeast construct show the localisation of the GFP signal. These images were further processed using surface profiling and line scans of signal intensity to better assess the distribution of GFP-AQP within the yeast cells. Yeast expressing GFP alone (Figure 2J), showed a uniform signal throughout the cell with the exception of the vacuole. The surface and line signal scans show a relatively equal distribution of intensity, consistent with cytosolic localisation. The fusion of NtAQPs to GFP resulted in the redistribution of GFP fluorescence to different yeast sub-cellular compartments including; PM, endoplasmic reticulum (ER), and/or the tonoplast (vacuolar membrane). The GFP-NtPIP1 fusions localised to the periphery of the cell and the ER. Although signal intensity in the periphery varied, likely due to co-localisation in peripheral ER, the signal remained continuous around the cell, consistent with PM integration (Figure $2 \mathrm{~A}-\mathrm{C}$ ). In addition to localisation in the yeast PM, images for NtPIP1;5s frequently contained bright spots in the periphery of the cell which are characteristic of ER localisation (Figure 2C). The NtPIP2 proteins integrated into the PM, with clearly defined peaks present in the line scans, but GFP signal also localised to the ER and faintly inside the vacuole (Figure 2E-D). NtNIP2;1s localised to the PM and ER, similar to that observed for the NtPIP1s (Figure 2F). NtNIP5;1 weakly localised to the PM and ER, with signal predominantly associated with integration into the tonoplast (Figure $2 \mathrm{G}$ ). The NtTIPs had strong signals distributed between the ER, tonoplast and notably the PM (Figure $2 \mathrm{H}-\mathrm{I}$ ). We confirmed PM integration for all NtAQP constructs tested. 


\section{Water permeability "Freeze-thaw" assay}

Growth curves of aqy1 aqy2 yeast expressing the Empty vector did not survive exposure to two freezethaw cycles, failing to grow after treatments (Figure 3A). By contrast, the growth aqy1 aqy2 yeast expressing NtPIP2;4s subjected to two freeze-thaw cycles was only slightly delayed compared to untreated yeast (Figure 3B). Substantial differences in growth were observed between treated and untreated yeast expressing either a particular NtAQP or empty vector (Figure 3C). Following exposure to two freeze-thaw cycles, the aqy1 aqy 2 yeast expressing the empty vector had only $2 \%$ growth relative to the untreated empty vector culture (Figure $3 C$ ). NtPIP2;4s and NtPIP2;5t expression resulted in the greatest yeast growth following the freeze-thaw treatments, achieving $70 \%$ of the untreated growth (Figure 3C). NtTIP1;1s and NtTIP2;5t grew $62 \%$ and $30 \%$, respectively, relative to untreated controls. Thus, four out of the nine NtAQPs tested were able to increase the permeability of the PM to water sufficiently to allow the yeast to survive two freeze-thaw treatments.

\section{$\mathrm{H}_{2} \mathrm{O}_{2}$ toxicity assay}

Dose-dependent differences upon exposure to increasing $\mathrm{H}_{2} \mathrm{O}_{2}$ treatments were observed in growth curves of yeast expressing the empty vector (Figure 4A) or a " $\mathrm{H}_{2} \mathrm{O}_{2}$-permeable" AQP (Figure 4B). Yeast expressing the empty vector and exposed to $0.25 \mathrm{mM}$ or $0.5 \mathrm{mM} \mathrm{H}_{2} \mathrm{O}_{2}$ treatments, had no significant reduction on growth relative to untreated yeast, while $1 \mathrm{mM} \mathrm{H}_{2} \mathrm{O}_{2}$ treatment caused a $37 \%$ decrease in growth relative to the untreated control (Figure $4 \mathrm{C}$ ). By contrast, growth was dramatically reduced in the presence of $0.25 \mathrm{mM} \mathrm{H}_{2} \mathrm{O}_{2}$ for yeast expressing NtPIP2;4s (growth reductions were $66 \%, 86 \%$ and $80 \%$ at $0.25 \mathrm{mM}, 0.5 \mathrm{mM}$ and $1 \mathrm{mM} \mathrm{H}_{2} \mathrm{O}_{2}$, respectively, Figure $4 \mathrm{C}$ ). Five NtAQPs increased yeast sensitivity to $\mathrm{H}_{2} \mathrm{O}_{2}$ exposure compared to empty vector, consistent with these AQPs being classified as $\mathrm{H}_{2} \mathrm{O}_{2}$ permeable: NtPIP2;4s, NtPIP2;5t, NtPIP1;1t, NtTIP1;1s and NtNIP2;1s. The lowest concentration of $\mathrm{H}_{2} \mathrm{O}_{2}$ that resulted in a significant reduction in growth compared to untreated was $0.25 \mathrm{mM}$ for NtPIP2;4s and NtPIP2;5t and 0.5mM for NtPIP1;1t, NtNIP2;1s and NtTIP1;1s.

\section{Boric acid toxicity assay}

Yeast expressing the empty vector (Figure 5A) or a "BA-permeable" AQP (Figure 5B) showed reduced growth in the presence of BA. Exposure of yeast expressing the empty vector control to $10 \mathrm{mM} B A$ did not impair growth (Figure $5 A$ and $5 C$ ). However, greater BA concentrations progressively reduced growth (by $33 \%$ and $64 \%$ at 20 and $30 \mathrm{mM} \mathrm{BA}$, respectively). Three phenotypes were observed across $\mathrm{NtAQP}$-expressing yeast exposed to $20 \mathrm{mM}$ and $30 \mathrm{mM}$ BA concentrations. The first phenotype displayed a 20-30\% reduction in growth relative to the empty vector yeast (NtTIP1;1s and NtTIP2;5t, Figure $5 \mathrm{C}$ ), consistent with these AQPs being classified as BA-permeable, enhancing a toxicity response. The second phenotype had a BA toxicity response that was within $10-20 \%$ of the empty vector (NtNIP2;1s, NtNIP5;1t and NtPIP1;5s, Figure 5C). Yeast expressing NtNIP2;1s had a significant reduction in growth at $10 \mathrm{mM}$ or $20 \mathrm{mM} \mathrm{BA}(p<0.05)$, suggesting moderate sensitivity to BA exposure. The third phenotype, observed in 4 of the 5 PIP-expressing yeasts (NtPIP1;1t, NtPIP1;3t, NtPIP2;4s, NtPIP2;5t), resulted in a greater tolerance to BA exposure (average growth $20 \%$ greater than empty vector at $20 \mathrm{mM}$ and $30 \mathrm{mM} \mathrm{BA}$, Figure $5 \mathrm{C}$ ). The reduced toxicity response associated with expressing any of these 4 NtPIPs could result from increased protein abundance in the PM reducing space available for free membrane diffusion of BA across the PM, thereby decreasing cell permeability to BA. Therefore, although NtPIP1;5s did not have a drastic decline in growth compared to the Empty vector control, growth was significantly reduced ( $25 \%$ at $20 \mathrm{mM} \mathrm{BA})$ compared to yeast expressing the other PIPs, likely due to boron permeability. 


\section{Urea growth-based assay}

Permeability of AQPs to urea was assessed by enhanced growth phenotypes. For ynvwl yeast, $12 \mathrm{mM}$ urea provided sufficient nitrogen for yeast cultures to reach a growth curve plateau within a $\sim 50$ hour incubation (Figure 6A-B, black lines). Growth curves of yeast expressing the empty vector (Figure 6A) or a "urea-permeable" AQP (Figure 6B) show that the expression of the latter enhanced yeast growth at low urea concentrations ( $2 \mathrm{mM}$ and $4 \mathrm{mM}$ urea). Yeast expressing the empty vector exhibited a linear growth response to increasing urea concentrations (Figure 6C). Expression of NtTIP1;1s, NtNIP2;1s or NtTIP2;5t resulted in a $50 \%$ growth enhancement at both $2 \mathrm{mM}$ and $4 \mathrm{mM}$ urea compared to yeast expressing an empty vector (Figure 6C). Growth responses for the other 6 NtAQPs (NtPIP1;1t, NtPIP1;5s, NtPIP1;3t, NtPIP2;4s, NtPIP2;5t, NtNIP5;1t), were similar to the yeast expressing the empty vector and these AQPs are presumed not permeable to urea.

\section{In planta sub-cellular localisation of tobacco AQPs}

Confocal images of root cortical cells from Arabidopsis plants expressing GFP-NtAQP constructs were obtained (Figure 7). To enhance interpretation, surface plots of a region of GFP intensity near the cell wall are shown at greater magnification (indicated by white dashed box). Organelle-specific marker lines for the plasma membrane, ER and tonoplast are shown in magenta (Figure 7A-C). We observed diversity in AQP membrane integration across the PIP, TIP and NIP subfamilies. The PIPs localised to the PM, with the PIP1s (PIP1;1t, PIP1;3t and PIP1;5s) appearing to have a weaker and more diffuse PM integration when compared to the PIP2s (PIP2;4s and PIP2;5t) which had a sharp and defined GFP signal around the cell's periphery (Figure 7D-H). The NtNIPs (NIP2;1s and NIP5;1t) also localised to the cell's periphery. However, their GFP signal was speckled in appearance with distinct localised spots of brighter fluorescence which are characteristic of ER localisation (indicated by white arrow on NIP2;1s surface plot profile of GFP intensity, Figure 7I-J). There was also a wider spread in GFP signal, arising from adjacent PM and ER. The localisation of NtTIPs (TIP1;1s and TIP2;5t) was consistent with integration in the tonoplast, showing a uniform yet diffuse localisation with a wavy topology; also denoted by the presence of internal membranes resembling transvacuolar strands ( $\mathrm{V}$, Figure $7 \mathrm{~K}-\mathrm{L}$ ).

\section{Protein modelling of aquaporin pores}

Tertiary structure homology modelling was used to compare pore diameter and the physico-chemical properties between the $9 \mathrm{NtAQPs}$ functionally characterised in this study. We made pair-wise comparisons between homology models based on the SoPIP2;1 (open; PDB: 2b5f.1.A) and AtTIP2;1 (PDB:5i32.1.A) crystal structure templates to ascertain the most appropriate (see Supplemental Methods S1).

Pore diameter profiles and physico-chemical characteristics of the NtAQPs across the different subfamilies were compared using the highest-confidence 3D homology models (Figure 8-9). All 5 pore diameter profiles for the NtPIPs closely overlapped (blue line, Figure 8A), with the SF region being the narrowest point along the pore (diameter of $2.4 \AA$ ). The SF residue composition of the NtPIPs was conserved, having Phe-His-Thr-Arg composition in Helix 2 (H2), Helix 5 (H5), Loop E position 1 (LE1) and Loop E position 2 (LE2), respectively. The TIPs also have a conserved pore diameter profile, with the SF region being the narrowest point (dark and light purple lines, Figure $8 \mathrm{~A}$ ). However, the pore shape of the NtTIPs was less undulating in comparison to the PIPs, with the SF region being wider $(2.75$ $\AA$ diameter) and the NPA region being slightly narrower ( $4.26 \AA$ diameter in TIPs vs. the $4.48 \AA$ of the PIPs; Figure S1 A, B). The SF residue composition differed between the $2 \mathrm{NtTIPs}$; with NtTIP1;1s having His- Ile- Ala- Val vs. NtTIP2;5t having His-lle-Gly-Arg at H2-H5-LE1-LE2 positions (Figure 8C). The NtNIPs that we characterised showed some variation in pore diameter profiles; with NtNIP2;1s having a SF diameter ranging from 3.5-4 $\AA$, vs. NtNIP5;1t's SF diameter of 2.6-3.5 $\AA$ (Figure 8A). The remainder of the pore toward the cytosolic side was similar in profile between these two genes. The differences in 
SF pore diameter between the NtNIPs were matched with variation in residue composition in this region, with NtNIP2;1s having SF residues: Gly-Ser-Gly-Arg and NtNIP5;1t: Ala-Ile-Ala-Arg at H2-H5LE1-LE2 positions respectively. The small size of the residues at the $\mathrm{H} 2$ and $\mathrm{H} 5$ position (Ser and Gly, respectively) in NtNIP2;1s, contribute to the larger pore diameter at this constriction site (Figure 8Civ). The composition of the NPA motifs (NPA1 and NPA2) is conserved across all of the NtPIPs, NtTIPs and NtNIP2;1s, with the exception of NtNIP5;1t which has NPS at NPA1 and NPV at NPA2 (Figure 8D).

We also characterised physico-chemical properties of the NtAQP pores (Figure 9). The NtPIPs have high homology in residue hydrophobicity and flexibility (Figure 9D). For both PIP1s and PIP2s, conserved regions around the cytosolic pore mouth and the narrowest part of the pore (the SF region) are lined with hydrophilic residues, whereas the remainder of the pore is lined with hydrophobic residues. The flexibility of the PIPs' pore lining residues was also conserved with a band of lowflexibility residues in their SF constriction region while the remainder of the pore having medium-high flexibility residues (Figure 9D). The pore profiles for NtTIPs were nearly cylindrical without the hourglass shape at the selectivity filter, being lined with mostly hydrophobic and less flexible residues (Figure 9B). Variation in flexibility was observed between the $2 \mathrm{NtNIPs,} \mathrm{with} \mathrm{pore} \mathrm{lining} \mathrm{residues} \mathrm{in}$ NtNIP5;1t being more flexible at the SF than in NtNIP2;1 (Figure 9C). The apoplastic pore entrance of NtNIP2;1 was very flexible and more hydrophilic than NtNIP5;1t (Figure 9C). All the NIPs and TIPs had low flexibility at the NPA central bottleneck region.

\section{Discussion}

NtAQP integration into the plasma membrane of yeast cells

A fundamental assumption in permeability assays is that the expressed AQP is present in the plasma membrane. We established this by using GFP translational fusions to visualise NtAQP subcellular localisation for each construct in yeast. Having confirmed that all AQP isoforms integrated into the PM in yeast (Figure 2), we could then infer that alterations to the growth of yeast upon exposure to specific substrates were due to AQP-related changes in PM permeability (Bienert and Chaumont 2014). Although we detected fluorescence consistent with incorporation of GFP tagged NtPIP1s in the PM of yeast (Fig. 2), there are several cases where PIP1 permeability to water has only been observed when PIP1 was co-expressed with a PIP2 (Xenopus oocytes, (Fetter et al. 2004), Yeast, (Groszmann et al. in preparation), reviewed in (Groszmann et al. 2017)). In future work, it would be useful to assess whether co-expression of PIP1s with PIP2s maximises detection of permeability to water or other substrates. Observing both GFP fluorescence consistent with localisation in the PM together with a positive increase in apparent permeability for one substrate enabled us to assign a 'non-permeable' result for other substrates for a given AQP. We therefore propose that confirmation of PM integration should be a necessary checkpoint when functionally testing AQPs in heterologous expression systems.

\section{Relating functional characterisation, sub-cellular localisation, and gene}

\section{expression of NtAQPs}

Having established that the expressed NtAQP were incorporated into the yeast PM, we present the combined permeability results, in planta sub-cellular localisation in Arabidopsis root cortical cells, gene expression localisation and protein modelling results in Table 1. The NtAQP data are placed in context with multiple permeabilities published for other AQPs. In planta subcellular localisation of the NtAQPs was consistent with their respective AQP subfamilies. 


\section{NtPIPs}

The PIP subfamily in plants generally has the largest number of isoforms (Anderberg et al. 2012). They are involved in plant water homeostasis through highly selective water transport activity, as well as facilitating diffusion of other small molecules such as glycerol, urea, BA, arsenous acid, $\mathrm{H}_{2} \mathrm{O}_{2}$, gases and ions (Bienert et al. 2018; Liu et al. 2020). Certain isoforms enhance permeability to multiple molecules e.g. AtPIP2;1 permeable to water, $\mathrm{H}_{2} \mathrm{O}_{2}, \mathrm{CO}_{2}$ and cations, with functionality dictated by its location within the plant, interacting proteins and associated post-translational modifications (Tyerman et al. 2021).

Both NtPIP2s tested were permeable to water and $\mathrm{H}_{2} \mathrm{O}_{2}$, with NtPIP2;4s seemingly more efficient at transporting $\mathrm{H}_{2} \mathrm{O}_{2}$ than PIP2;5t. $\mathrm{H}_{2} \mathrm{O}_{2}$ permeability was predicted for these two PIP2s by Ahmed et al. (2020) based on substrate specific signature sequences (Azad et al. 2016) (see (Groszmann et al. 2021) to convert between equivalent NtAQP gene names). None of the three NtPIP1s examined here enhanced permeability to water, but were permeable to $\mathrm{H}_{2} \mathrm{O}_{2}$ (NtPIP1;1t) or BA (NtPIP1;5s) (Table 1). As permeability to $\mathrm{H}_{2} \mathrm{O}_{2}$ and BA was not predicted for these PIP1s by Ahmed et al. (2020), subtle sequence differences or pore dynamics of these isoforms clearly can modify an AQP's preferential transport to a given substrate (Qiu et al. 2020). Various PIP1 and 2 isoforms from other species are permeable to BA (e.g. ZmPIP1;1 (Dordas et al. 2000), VvPIP1;4 and VvPIP2;3 (Sabir et al. 2014), HvPIP1;3 and 1;4 (Fitzpatrick and Reid 2009), OsPIP2;4 and 2;7 (Kumar et al. 2014)). NtPIP1;5s has been reported to have a low permeability to $\mathrm{H}_{2} \mathrm{O}_{2}$ using a fluorescence dye-based assay (NavarroRóDenas et al. 2015), but as we could not detect permeability to $\mathrm{H}_{2} \mathrm{O}_{2}$, this needs to be confirmed using co-expression of both PIP1 and PIP2.

Combining substrate permeabilities with in planta subcellular localisation and tissue specific expression analysis helps elucidate possible physiological roles for these NtAQPs. The results implicate $N t P I P 2 ; 4 s$ and NtPIP2;5t having roles in regulating water transport across cell membranes in roots and leaves, respectively. Three of the NtPIPs tested here were permeable to $\mathrm{H}_{2} \mathrm{O}_{2}$, so are likely to be involved with ROS signalling in response to stress (Hachez et al. 2006) by facilitating $\mathrm{H}_{2} \mathrm{O}_{2}$ diffusion between cells. NtPIP1;5s (NtAQP1) was the first plant AQP shown to permeate $\mathrm{CO}_{2}$ (Uehlein et al. 2003), facilitating diffusion of $\mathrm{CO}_{2}$ into the chloroplast during photosynthesis (Flexas et al. 2006). We suggest NtPIP1;5s might have an additional role in boron uptake and distribution throughout the plant (expressed in roots, stems, leaves and flowers), similar to the functional roles reported for other boron-permeable PIPs, HvPIP1;3 and HvPIP1;4 (Fitzpatrick and Reid 2009) and OsPIP2;4 and OsPIP2;7 (Kumar et al. 2014). The boron-transport capability of the PIPs, and their narrow inflexible pore constriction site (2.4A SF size vs. $5.14 \AA$ Aize of BA) suggests that permeability of this substrate could potentially occur through the tetrameric central pore.

\section{NtTIPs}

The TIP subfamily regulates the diffusion of water, ammonia, urea and metalloids across the tonoplast (Maurel et al. 1993; Loqué et al. 2005; Liu et al. 2003). Five specialised TIP subgroups have evolved in higher plants (TIP1-TIP5), differing in ar/R filter (SF) composition and substrate specificities (Anderberg et al. 2012; Kirscht et al. 2016). Exemplars from both TIP1 (NtTIP1;1s) and TIP2 (NtTIP2;5t) subgroups localised to the tonoplast in planta, where they could increase permeability to water, BA and urea. NtTIP1;1s was also moderately permeable to $\mathrm{H}_{2} \mathrm{O}_{2}$ but NtTIP2;5t was not. The substrate permeabilities observed here for NtTIP1;1s exactly match those for ZmTIP1;1 (Fox et al. 2017).

The SF region of the NtTIPs was found to be wider than that of the NtPIPs. An extended selectivity filter has been characterised for the TIP subfamily, containing an additional contact residue in Loop C of the AQP monomer (Kirscht et al. 2016), with NtTIP1;1s and NtTIP2;5t having a Phe and His at this position, respectively (De Rosa et al. 2020). The NtTIP1;1 SF composition of a Phe in Loop C and a Val in Loop E2 creates a more hydrophobic environment in the SF compared to that of NtTIP2;5s which 
has His and $\mathrm{Arg}$ in the same two positions respectively. This results in TIP1;1s being more hydrophobic than TIP2;5t in its constriction region (Figure 9B). While Ahmed et al. (2020) correctly predicted TIP1;1s should be permeable to $\mathrm{H}_{2} \mathrm{O}_{2}$ and urea, our results did not match their prediction for TIP2;5t for $\mathrm{H}_{2} \mathrm{O}_{2}$ and ammonia. Instead we found TIP2;5t was permeable to urea, but not $\mathrm{H}_{2} \mathrm{O}_{2}$. The differences in SF composition and hydrophobicity between NtTIP1;1s and NtTIP2;5t might explain their divergences in in permeability to $\mathrm{H}_{2} \mathrm{O}_{2}$ (Table 1) and potentially also ammonia (Kirscht et al. 2016). NtTIP1;1s is expressed in leaves and flowers whereas NtTIP2;5t is predominantly expressed in the roots (having low expression in leaves and flowers). Root-specific gene expression of TIP2 isoforms was also observed in closely related gene ortholog, tomato TIP2;5 (De Rosa et al. 2020) and more distantly related maize TIP2s (Fox et al. 2017), indicating potential conservation of function of TIP2s across closely related and diverse species. The proposed functional roles for NtTIP1;1s and NtTIP2;5t include the loading and unloading of urea from vacuolar storage, the storage and translocation of boron, and equilibration of water in tissues where they are expressed (Maurel et al. 2015). Furthermore, NtTIP1;1s could also be involved in ROS signalling in response to stress, as it has been suggested that TIPs are involved in cellular detoxification of $\mathrm{H}_{2} \mathrm{O}_{2}$ (Bienert and Chaumont 2014).

\section{NtNIPs}

NIP aquaporins are known to facilitate the transport of small uncharged solutes, such as glycerol, urea and metalloids (Wallace et al. 2006). NIPs have a more hydrophobic ar/R selectivity filter, which reduces water permeability in favour of other substrates such as ammonia, urea and metalloids (Wu and Beitz 2007; Hove and Bhave 2011). There are three sub-classes (NIP I-III), based on ar/R selectivity filter and NPA motif composition (Mitani et al. 2008). A representative from each of NIP II (NtNIP5;1t) and NIP III (NtNIP2;1s) sub-classes were characterised here. GFP tagging demonstrated that both NIP2;1s and NIP5;1s were incorporated into the PM as well as accumulating in the ER in planta. The integration in the plant cell PM (versus tonoplast localisation of the TIPs) implies transport of solutes in and out of cells, rather than simply storage/translocation from the vacuole.

NIP II aquaporins tend to have a larger pore diameter than those found in the NIP I sub class, having a substitution of the highly conserved and bulky Trp at the ar/R H2 position for a smaller Ala (Wallace and Roberts 2004). NtNIP5;1t had a wider pore diameter compared to that of the tobacco PIP and TIP isoforms (Figure 8). NIP II aquaporins have been shown to permeate BA, glycerol and urea, together with reduced water permeability compared to the NIP Is (Wallace et al. 2006; Takano et al. 2006; Hanaoka et al. 2014; Tanaka et al. 2008). By contrast, while NtNIP5;1t enhanced permeability to BA, it did not enhance permeability to urea despite their similar size (Table 1) NtNIP5;1t expression is highly targeted to young flowers and could be involved in boron redistribution during flower development, similar to the orthologous gene in Arabidopsis (AtNIP5;1), which has an established role in boron transport and flower development (Takano et al. 2006). Notably, expression of NIP II isoforms, AtNIP5;1 (in flowers) and AtNIP6;1 (in basal shoot), is induced in boron-limiting conditions (Takano et al. 2006; Tanaka et al. 2008). The variation in expression patterns (localisation and stressresponsiveness) reported for PIPs vs. NIP Ils, allude to differences in physiological relevance of their boron transport in planta. PIPs could mediate a broad boron uptake and distribution (Fitzpatrick and Reid 2009), however their co-function as water channels subjects them to tight regulation (Chaumont and Tyerman 2014). Boron-permeable PIPs would also provide tolerance to boron toxicity by enabling efflux of excess boron from roots and shoots (Kumar et al. 2014; Kumar et al. 2018). Unlike the PIPs, water-tight NIP Ils enable highly targeted boron transport in boron-limiting conditions, and are downregulated in boron sufficient concentrations (Takano et al. 2006; Chaumont and Tyerman 2017).

NIP III aquaporins (such as NtNIP2;1s) are characterised by an ar/R filter composed of smaller residues (Gly-Ser-Gly-Arg), resulting in an even wider, flexible and more hydrophilic SF (Bansal and Sankararamakrishnan 2007; Mitani-Ueno et al. 2011). Our 3D homology modelling showed that NtNIP2;1s indeed has a wider pore than all the other NtAQP isoforms characterised (Figure 8), 
consistent with this sub-class being permeable to larger substrates, such as silicic acid $(4.38 \AA$ diameter) and lactic acid (Mitani-Ueno et al. 2011). Unlike NIP5;1t, NIP2;1s was permeable to multiple substrates, urea, BA and $\mathrm{H}_{2} \mathrm{O}_{2}$ (low), implicating this NIP isoform in multiple functional roles.

NIP III isoforms occur widely among Graminae, but are not found in all dicots (e.g. absent in Arabidopsis), with evidence suggesting their principal role as facilitators of silicon uptake in plants (Chaumont and Tyerman 2017). In addition to silicon, ZmNIP2;1 (NIP III) is also permeable to water, urea, BA and $\mathrm{H}_{2} \mathrm{O}_{2}$ (low) (Fox et al. 2017), suggesting some functional homology in the maize and tobacco isoforms. Expression of NtNIP2;1 is restricted to young flowers where it is likely to be involved in strategic translocation of small molecules in this target tissue.

\section{Conclusions}

This study characterised a diverse set of isoforms in the tobacco AQP family assessing permeability to key solutes for plant growth: water, $\mathrm{H}_{2} \mathrm{O}_{2}$, urea and boric acid. The functional diversity observed between the NtAQP isoforms highlights complexity in assigning in planta function to specific isoforms, with monomeric pore shape/size, SF and NPA motifs alone insufficient to comprehensively predict their transport capabilities.

We observed permeability to each of the substrates tested across three largest AQP subfamilies, with substrate selectivity ranging from none to all substrates tested, indicative of specific or broad functional roles. By using isoforms with sequence homology to well characterised AQPs from other species (NtTIP2;5t to AtTIP2;1 and NtNIP5;1t to AtNIP5;1), we could correlate sequence similarity to functional homology, as well as identify novel permeating substrates to extend their transport profiles.

We propose that testing transport capability to several substrates could be used to assign in planta roles to multifunctional aquaporins.

\section{Methods}

\section{Generation of NtAQP phylogeny}

MUSCLE-aligned nucleotide sequences identified in De Rosa et al. 2020 and Ahmed et al. 2020, (consensus NtAQP family in (Groszmann et al. 2021)), were used to construct a phylogenetic tree using neighbour-joining method (pair-wise deletion; bootstrap=1000) in MEGA7 software (Kumar et al. 2016).

\section{Generation of NtAQP expression constructs and transformation into yeast}

Native sequences of NtAQPs sequences, NtPIP1;1t (BK011393), NtPIP1;3t (BK011396), NtPIP1;5s (BK011398), NtPIP2;4s (BK011406), NtPIP2;5t (BK011409), NtTIP1;1s (BK011426), NtTIP2;5t (BK011440), NtNIP2;1s (BK011379), NtNIP5;1t (BK011387) were commercially synthesised in Gateway-enabled destination vectors. Entry vectors were cloned into three destination vectors from (Alberti et al. 2007): pRS423-GPD with a Histidine3 (HIS3) marker gene for yeast selection, and pRS423-GPD-ccdB-ECFP and pRS426-GPD-eGFP-ccdB both containing Uracil3 (URA3) yeast selection gene. Yeast expression vectors were transformed in respective yeast strains required for functional assays (described below), using the "Frozen-EZ yeast Transformation Kit II" (Zymo Research, Los Angeles, USA). Transformed colonies were grown in Yeast Nitrogen Base, YNB, media (Standard drop out, DO, -URA or -HIS) and spotted on agar YNB (DO -URA or -HIS) selection plates for incubation at $30^{\circ} \mathrm{C}$ for 2 days, then stored at $4^{\circ} \mathrm{C}$. Spotted plates were used for the starting cultures of functional assays. 


\section{Confirming plasma membrane integration of NtAQPs in yeast cells}

We assessed NtAQP subcellular localisations in yeast cells with AQP-GFP translational fusions to confirm incorporation of the expressed AQP in the yeast plasma membrane (PM). Tobacco AQP:GFP translational fusions were generated via gateway cloning of pUC57 entry vectors with NtAQP coding sequences into pRS426-GPD-EGFP-ccdB yeast expression vector (Alberti et al. 2007); producing Nterminal GFP::NtAQP fusion proteins driven by the constitutive GPD promoter. The GFP-only yeast expression was obtained using the empty vector (no GOI fusion), with eGFP alone constitutively expressed via the GPD promoter. Yeast was grown overnight in $2 \mathrm{~mL}$ YNB (-URA) $\left(\mathrm{OD}_{600} 1-1.5\right)$. $1 \mathrm{~mL}$ aliquots of overnight cultures were sub-cultured and grown 3-4 hours in $2 \mathrm{~mL}$ of fresh YNB (-URA) media, to ensure imaging of newly formed cells. Yeast $(10 \mu \mathrm{L})$ was mounted on a polysine slide with a coverslip sealed with nail polish. Yeast cells were visualised with a Zeiss LSM 780 Confocal microscope using a 40x oil immersion objective (1.2 NA). Light micrographs of yeast cells were acquired using Differential Interference Contrast (DIC), with GFP fluorescence captured using excitation at $488 \mathrm{~nm}$ and emission detection across the 490-526 nm range. Images were processed using Fiji (ImageJ) software (Schindelin et al. 2012).

\section{Assessing water, $\mathrm{H}_{2} \mathrm{O}_{2}$, boric acid and urea permeability using high-throughput yeast-based \\ assays}

Yeast-based microculture assays (Groszmann et al. in preparation), were used to test membrane permeability to specific substrates associated with the expression of foreign AQPs which resulted in enhanced or impaired growth. $\mathrm{H}_{2} \mathrm{O}_{2}, \mathrm{BA}$ and Urea treatments were previously optimised in (Groszmann et al. in preparation). Yeast growth was monitored using a SPECTROStar nano absorbance microplate reader (BMG Labtech, Germany) at 10-20 minute intervals over 42 to 60 hours. Data collection and processing was consistent between each growth or toxicity-based assay.

Water permeability. We used the aqy1 aqy2 yeast strain (null aqy1 aqy2; background $\Sigma 1278 \mathrm{~b}$; genotype: Mat $\alpha$; leu2::hisG; trp1::hisG, his3::hisG; ura352 aqy1D::KanMX aqy2D::KanMX, provided by Peter Dahl of the S. Hohmann lab) (Tanghe et al. 2002) exploiting the property of yeast cells that show increased freezing tolerance when they express functional water AQPs (Deshmukh et al. 2016). Yeast expressing NtAQPs and the empty vector were grown for $24-28$ hours $\left(\mathrm{OD}_{650}\right.$ of $\left.0.5-1\right)$ in $1.25 \mathrm{~mL}$ YNB(-HIS), at $30^{\circ} \mathrm{C}$, shaking at $250 \mathrm{rpm}$. Cultures were diluted to $0.6 \times 10^{7}$ cells $/ \mathrm{mL}$ in YPD medium and incubated at $30^{\circ} \mathrm{C}$ for 60 mins. $250 \mu \mathrm{L}$ of each culture was aliquoted to 2 Eppendorf tubes; one tube was placed on ice (untreated control) the other was used for two 'Freeze-thaw' cycles. Each 'Freeze thaw' cycle consisted of yeast culture aliquots being frozen in liquid nitrogen for 30 seconds, and thawed in a water bath at $30^{\circ} \mathrm{C}$ for 20 mins. For each construct, 'Untreated' and 'Treated' yeast were transferred into a 96 well plate $(200 \mu \mathrm{L}$ aliquots) for growth monitoring.

$\mathrm{H}_{2} \mathrm{O}_{2}$ permeability was assessed using a reactive oxygen species (ROS) hypersensitive yeast strain, $\Delta s k n 7$ (null skn7; background BY4741 genotype: Mat $\alpha$; his3 $\Delta 1$ leu2 $\Delta 0$ met15 $\Delta 0$ ura3 $\Delta 0 \Delta$ SKN7). Obtained from ATCC) (Bienert et al. 2007; Halliwell and Gutteridge 2015; Lee et al. 1999). Yeast's survival was further compromised if AQPs facilitated the diffusion (and accumulation) of $\mathrm{H}_{2} \mathrm{O}_{2}$ into the cell, which enhanced the toxicity response. $\Delta s k n 7$ yeast expressing NtAQPs and Empty vector were grown and diluted to $0.6 \times 10^{7}$ cells $/ \mathrm{mL}$ as per the Freeze-thaw assay (above). $200 \mu \mathrm{L}$ microcultures of each NtAQP/Empty vector were distributed in 96-well plates with $190 \mu \mathrm{L}$ of yeast and $10 \mu \mathrm{L} \mathrm{H}_{2} \mathrm{O}_{2}$ treatments: $0 \mathrm{mM} /$ water, $0.25 \mathrm{mM}, 0.5 \mathrm{mM}$ and $1 \mathrm{mM} \mathrm{H}_{2} \mathrm{O}_{2}$.

Boric acid (BA) toxicity assays were used to assess AQP permeability to BA. Enhanced BA diffusion into yeast expressing AQP lead to greater sensitivity to BA treatments. aqy1 aqy 2 yeast expressing NtAQPs or Empty vector were grown and diluted to $0.6 \times 10^{7}$ cells $/ \mathrm{mL}$. $200 \mu \mathrm{L}$ microcultures each NtAQP/Empty vector were distributed in 96 -well plates with $180 \mu \mathrm{L}$ of yeast and $20 \mu \mathrm{L}$ BA treatments: $0 \mathrm{mM} /$ water, $10 \mathrm{mM}, 20 \mathrm{mM}$ and $30 \mathrm{mM}$ BA. 
Urea transport capability. ynvwl yeast (null dur3; background 223346 c; genotype: Mat $\alpha, \Delta$ ura3, $\Delta$ dur3, provided by Patrick Bienert of the Nicolaus von Wirén lab) is limited in growth due to a deletion of the DUR3 urea transporter (Liu et al. 2003). Expression of urea-permeable AQPs in ynvwl yeast provided a growth advantage when exposed to media containing urea as the sole nitrogen source. ynvwl yeast spots were resuspended in $1.25 \mathrm{~mL}$ of Yeast Basic media (YB, culture medium without nitrogen source) with $2 \%$ Glucose. Yeast cultures were diluted to $1.2 \times 10^{7}$ cells $/ \mathrm{mL}$. $200 \mu \mathrm{L}$ microcultures for each NtAQP/Empty vector construct were distributed in 96-well plates with $190 \mu \mathrm{L}$ of yeast and $10 \mu \mathrm{L}$ urea treatments: $0 \mathrm{mM} /$ water, $1 \mathrm{mM}, 4 \mathrm{mM}$ and $12 \mathrm{mM}$ urea.

The yeast microculture $\mathrm{OD}_{650}$ readings for the water, $\mathrm{H}_{2} \mathrm{O}_{2}, \mathrm{BA}$ and urea assay were processed using a method developed by (Groszmann et al. in preparation). Growth curves were integrated using the natural log of $O D_{650} /$ initial $O D_{650}\left(\operatorname{Ln}\left(O D_{t} / O D_{i}\right)\right.$ vs time) up to a time when the growth rate of the Untreated culture had declined to $5 \%$ of its maximum. Area Under the Curve (AUC), was calculated as a proxy that captured the potential growth characteristics affected, regardless of the treatment, in a single parameter.

\section{Characterising in planta subcellular localisation of NtAQPs}

Tobacco AQP-GFP constructs were generated via Gateway cloning of NtAQP coding sequences from pZeo entry vectors into the pMDC43 destination vector (Curtis and Grossniklaus 2003); N-terminal GFP-NtAQP fusion proteins were driven by the constitutive 2x35S CaMV promoter. Arabidopsis transgenic lines were generated via agrobacterium (GV3101) floral dipping transformation method (Clough and Bent 1998). Seeds were liquid sterilised, washed and sown on Gamorg's B5 medium (0.8\% Agar, hygromycin). 8-day-old Arabidopsis seedlings were gently removed from the agar, mounted in phosphate Buffer $\left(100 \mathrm{mM} \mathrm{NaPO}_{4}\right.$ buffer, $\mathrm{pH}$ 7.2) on a standard slide, covered with coverslip, and visualised with a Zeiss LSM 780 Confocal microscope using a 40x water immersion objective (1.2 NA). Light micrographs of cortical cells in the root elongation zone were visualised using Differential Interference Contrast (DIC), GFP fluorescence was captured using excitation at $488 \mathrm{~nm}$ and emission detection across 490-526 nm. Autofluorescence was detected across 570-674 nm and excluded from GFP detection channel. Images were processed using Fiji (ImageJ) software (Schindelin et al. 2012). Organelle-specific marker lines, established in Nelson et al. (2007) and previously published in De Rosa et al. (2020), were used to guide our interpretation of AQP subcellular localisations.

\section{D protein homology modelling and characterisation of NtAQP pores}

We generated 3D models of the 9 NtAQPs functionally characterised in this study. The Spinach PIP2;1 resolved crystal structure (Törnroth-Horsefield et al. 2006) and Arabidopsis TIP2;1, PDB:5i32.1.A (Kirscht et al. 2016), were used as templates for our modelling analyses. See Supplemental Methods S1 for more background detail.

\section{Acknowledgements}

We thank Rosemary White from CSIRO for providing seeds of the Tonoplast:GFP and ER:GFP marker lines (Nelson et al. 2007). The authors acknowledge the facilities and the scientific and technical assistance (Darryl Webb) of Microscopy Australia at the Advanced Imaging Precinct at the Australian National University; a facility funded by the ANU, and State and Federal Governments of Australia. We acknowledge that National Collaborative Research Infrastructure Strategy (NCRIS) of the Australian Government, providing The Australian National University with the growth facilities utilised as part of the Australian Plant Phenomics Facility. 
507

508

509

510

511

512

513

514

515

516

517

518

519

520

521

522

523

524

525

526

527

528

529

530

531

532

533

534

535

536

537

538

539

540

541

Table 1. Summary of NtAQP properties and other AQP isoforms characterised in other studies. Permeability to water, $\mathrm{H} 2 \mathrm{O} 2$, boric acid and urea, molecular diameter (Å) shown in parenthesis; in planta sub-cellular localisation in root cortical cells, Selectivity filter (SF), pore diameter ( $(\AA)$ and gene expression localisations (reported in De Rosa et al. 2020). Red tick $(\checkmark)$ denotes a positive permeability for a specific substrate with the number of ticks indicating the magnitude of phenotypic responses. One, two and three ticks represent a small, medium and large effect, respectively. $C$ denotes water permeable when PIP2 co-expressed with PIP1 and permeability for other substrates when coexpressed. Cross $(\boldsymbol{x})$ denotes a negative permeability. Blanks indicate no data is available. Three subcellular localisations for the NtAQPs were tested: plasma membrane (PM), endoplasmic reticulum (ER) and tonoplast.

(1)

1

3

4


bioRxiv preprint doi: https://doi org/10.1101/2021.03.26.437249; this version posted March 28, 2021. The copyright holder for this preprint (which was not certified by peer review) is the author/funder, who has granted bioRxiv a license to display the preprint in perpetuity. It is made available under aCC-BY-NC-ND 4.0 International license.

\begin{tabular}{|c|c|c|c|c|c|c|c|c|}
\hline & $\begin{array}{l}\text { Water } \\
(2.75 \AA)\end{array}$ & $\begin{array}{l}\mathbf{H}_{2} \mathbf{O}_{2} \\
(2.8 \AA)\end{array}$ & $\begin{array}{c}\text { Boric } \\
\text { Acid } \\
(5.14 \AA)\end{array}$ & $\begin{array}{c}\text { Urea } \\
(5.22 \AA)\end{array}$ & $\begin{array}{c}\text { In planta } \\
\text { subcellular } \\
\text { localisation }\end{array}$ & $\begin{array}{c}\text { SF pore } \\
\text { diameter } \\
(\AA)\end{array}$ & $\begin{array}{l}\text { Expression } \\
\text { localisation }\end{array}$ & Ref. \\
\hline ZmPIP1 & C & $x$ & $x$ & $x$ & & & & $\begin{array}{c}\text { (Fox et al. } \\
2017)\end{array}$ \\
\hline AtPIP1;1 & C & $\checkmark$ & $\checkmark \checkmark$ & $x$ & & & & $\begin{array}{l}\text { (Groszmann } \\
\text { et al. in } \\
\text { preparation) }\end{array}$ \\
\hline NtPIP1;1t & $x$ & $\checkmark$ & $x$ & $x$ & PM & 2.40 & Roots & \\
\hline NtPIP1;3t & $x$ & $x$ & $x$ & $x$ & PM & 2.40 & Whole plant & \\
\hline AtPIP1;4 & C & $\checkmark \checkmark$ & $x$ & $x$ & & & & $\begin{array}{l}\text { (Groszmann } \\
\text { et al. in } \\
\text { preparation) }\end{array}$ \\
\hline NtPIP1;5s & $x$ & $x$ & $\checkmark$ & $x$ & PM & 2.40 & $\begin{array}{l}\text { Leaves, } \\
\text { stem, roots, } \\
\text { flowers }\end{array}$ & \\
\hline AtPIP2;4 & $\checkmark$ & $\checkmark$ & $x$ & $x$ & & & & $\begin{array}{l}\text { (Groszmann } \\
\text { et al. in } \\
\text { preparation) }\end{array}$ \\
\hline NtPIP2;4s & $\checkmark \checkmark$ & $\checkmark \checkmark \checkmark$ & $x$ & $x$ & PM & 2.40 & $\begin{array}{c}\text { Roots, } \\
\text { flowers (low) }\end{array}$ & \\
\hline NtPIP2;5t & $\checkmark \checkmark$ & $\checkmark \checkmark$ & $x$ & $x$ & PM & 2.40 & Leaves & \\
\hline ZmPIP2;5 & $\checkmark \checkmark$ & $\checkmark \checkmark$ & - & - & & & $\begin{array}{l}\text { Roots, stem, } \\
\text { leaves, }\end{array}$ & $\begin{array}{c}\text { (Fox et al. } \\
2017)\end{array}$ \\
\hline AtPIP2;7 & $\checkmark \checkmark$ & $\checkmark \checkmark$ & $\checkmark$ & $x$ & & & & $\begin{array}{l}\text { (Groszmann } \\
\text { et al. in } \\
\text { preparation) }\end{array}$ \\
\hline ZmNIP1;1 & $\checkmark \checkmark$ & $\checkmark$ & $\checkmark$ & $x$ & & & & $\begin{array}{c}\text { (Fox et al. } \\
2017 \text { ) }\end{array}$ \\
\hline AtNIP1;2 & & $\checkmark$ & & & & & & $\begin{array}{l}\text { (Dynowski et } \\
\text { al. 2008) }\end{array}$ \\
\hline ZmNIP2;1 & $\checkmark \checkmark$ & $\checkmark$ & $\checkmark \checkmark$ & $\checkmark \checkmark$ & & & & $\begin{array}{c}\text { (Fox et al. } \\
2017)\end{array}$ \\
\hline NtNIP2;1s & $x$ & $\checkmark$ & $\checkmark$ & $\checkmark \checkmark$ & $\mathrm{ER}+\mathrm{PM}$ & 3.50 & $\begin{array}{l}\text { Young } \\
\text { flowers }\end{array}$ & \\
\hline AtNIP5;1 & $\checkmark$ & & $\checkmark \checkmark$ & & & & & $\begin{array}{l}\text { (Takano et } \\
\text { al. 2006; } \\
\text { Wang et al. } \\
\text { 2017) } \\
\end{array}$ \\
\hline NtNIP5;1t & $x$ & $x$ & $\checkmark$ & $x$ & $\mathrm{ER}+\mathrm{PM}$ & 3.26 & $\begin{array}{l}\text { Young } \\
\text { flowers }\end{array}$ & \\
\hline AtNIP6;1 & $x$ & & & $\checkmark \checkmark$ & & & & $\begin{array}{l}\text { (Wallace and } \\
\text { Roberts } \\
\text { 2005) } \\
\end{array}$ \\
\hline AtTIP1;1 & $\checkmark \checkmark$ & $\checkmark \checkmark$ & & & & & & $\begin{array}{c}\text { (Bienert et al. } \\
2007)\end{array}$ \\
\hline NtTIP1;1s & $\checkmark \checkmark$ & $\checkmark$ & $\checkmark \checkmark$ & $\checkmark$ & Tonoplast & 2.75 & $\begin{array}{l}\text { Leaves, } \\
\text { flowers }\end{array}$ & \\
\hline ZmTIP1;1 & $\checkmark \checkmark$ & $\checkmark \checkmark$ & $\checkmark \checkmark$ & $\checkmark \checkmark$ & & & & $\begin{array}{c}\text { (Fox et al. } \\
2017)\end{array}$ \\
\hline AtTIP1;3 & $\checkmark \checkmark$ & & $x$ & $\checkmark \checkmark$ & & & & $\begin{array}{l}\text { (Soto et al. } \\
2008 \text { ) }\end{array}$ \\
\hline AtTIP2;1 & $\checkmark$ & & & $\checkmark$ & & & & $\begin{array}{c}\text { (Liu et al. } \\
2003 \text { ) }\end{array}$ \\
\hline NtTIP2;5t & $\checkmark$ & $x$ & $\checkmark \checkmark$ & $\checkmark$ & Tonoplast & 2.75 & $\begin{array}{c}\text { Roots, } \\
\text { leaves (low), } \\
\text { flowers (low) }\end{array}$ & \\
\hline
\end{tabular}


Figure 1. Phylogeny of the NtAQP family, highlighting genes selected for functional characterisation in this study. The phylogenetic tree was generated using the neighbour-joining method from MUSCLE aligned protein sequences. Confidence levels (\%) of branch point generated through bootstrapping analysis ( $n=1000$ ). Red arrows point to PIP (PIP1;1t, PIP1;3t, PIP1;5s, PIP2;4s, PIP2;5t), TIP (TIP1;1s, TIP2;5t) and NIP (NIP2;1s and NIP5;1t) isoforms functionally characterised in this study.

Figure 2. Subcellular localisation of GFP tagged aquaporins expressed in yeast. Confocal microscopy images of yeast expressing GFP::NtAQP fusions of A. NtPIP1;1t, B. NtPIP1;3t, C. NtPIP1;5s, D. NtPIP2;4s, E. NtPIP2;5t, F. NtNIP2;1s, G. NtNIP5;1t, H. NtTIP1;1s, I. NtTIP2;5t and J. Free GFP localisation. For each construct we report a Brightfield + GFP overlay image of a yeast cell; a GFP only image; a surface plot profile of GFP signal intensity at the imaged focal plane; and a line scan of signal intensity traversing the cell (indicated by white arrow in GFP only image). Grey shading in GFP signal line scan corresponds to regions which align with the plasma membrane (PM). PM, endoplasmic reticulum (ER) and vacuole (V) are labelled. Scale bar $2 \mu \mathrm{m}$.

Figure 3. NtAQP water permeability assessed with the 'Freeze-thaw' assay. Yeast growth curves, $\operatorname{Ln}\left(O D / O D_{i}\right)$ vs. time, of aqy1 aqy2 yeast expressing $\mathbf{A}$. Empty vector control or $\mathbf{B}$. a freeze-thaw tolerant AQP (NtPIP2;4s), exposed to freeze-thaw treatments. Growth was assessed from the area under the curves $(A \cup C)$ until the vertical dashed lines. C. Yeast culture growth following the freezethaw treatment ( $A \cup C$ relative to untreated yeast control) for aqy1 aqy2 yeast expressing an Empty vector or one of the 9 NtAQPs. Asterisks denote significantly greater growth following the freeze thaw treatment compared against Empty vector from an ANOVA with Fishers LSD test: “**” $p<0.01$ and “***” $\mathrm{p}<0.001, \mathrm{~N}=6$, Error bars $=\mathrm{SE}$.

Figure 4. $\mathrm{NtAQP} \mathrm{H}_{2} \mathrm{O}_{2}$ permeability assay. Yeast growth curves, $\operatorname{Ln}\left(O D / O D_{i}\right)$ vs. time, of snk7 yeast expressing A. Empty vector control or B. an $\mathrm{H}_{2} \mathrm{O}_{2}$-sensitive AQP (NtPIP2;4s), exposed to $0.25 \mathrm{mM}$, $0.5 \mathrm{mM}$ and $1 \mathrm{mM} \mathrm{H}_{2} \mathrm{O}_{2}$ treatments. Growth was assessed from the area under the curves (AUC) until the vertical dashed lines. C. Yeast culture growth relative to 'Untreated' control (AUC relative to untreated), for skn7 yeast expressing an Empty vector or one the 9 NtAQPs. Asterisks denote One-way ANOVA with Fishers LSD test results comparing $\mathrm{H}_{2} \mathrm{O}_{2}$-treated yeast growth against Empty vector; "*" $\mathrm{p}<0.05, " * * " \mathrm{p}<0.01$ and $" * * * " \mathrm{p}<0.001$. $\mathrm{N}=6$, Error bars $=\mathrm{SE}$.

Figure 5. Boric acid permeability assay for yeast expressing NtAQPs. Yeast growth curves, $\operatorname{Ln}\left(O D / O D_{i}\right)$ vs. time, of aqy1 aqy2 yeast expressing $\mathbf{A}$. Empty vector control or $\mathbf{B}$. a boric acid-sensitive AQP (NtTIP1;1s), exposed to $10 \mathrm{mM}, 20 \mathrm{mM}$ and $30 \mathrm{mM}$ boric acid treatments. Growth was assessed from the area under the curves $(A \cup C)$ until the vertical dashed lines. $C$. Yeast culture growth relative to untreated control (AUC) of aqy1 aqy2 yeast expressing either an Empty vector or one of the 9 $\mathrm{NtAQPs}$ exposed to boric acid. Asterisks denote growth that was significantly different to Empty Vector using a One-Way ANOVA with Fishers LSD test; “*” $p<0.05$, “**” $p<0.01$ and “***” $p<0.001, N=6$, Error bars=SE.

Figure 6. Urea permeability assays for yeast expressing NtAQPs. Yeast growth curves, $\operatorname{Ln}\left(O D / O D_{i}\right)$ vs. time, of ynvwl yeast expressing A. Empty vector control or B. a urea-permeable AQP (NtNIP2;1s), exposed to $0 \mathrm{mM}, 2 \mathrm{mM}, 4 \mathrm{mM}$ or $12 \mathrm{mM}$ urea treatments. Growth was assessed from the area under the curves (AUC) until the vertical dashed lines. C. AUC relative to $0 \mathrm{mM}$ Urea treatment of ynvwl yeast expressing either an Empty vector or one of the 9 screened NtAQPs. Asterisks denote growth that was significantly greater than Empty vector using a One-way ANOVA with Fishers LSD test, “*” $p<0.05$, “**” $p<0.01$ and “***" $p<0.001 . \mathrm{N}=6$, Error bars=SE.

Figure 7. In planta sub-cellular localisation of NtAQPs. Confocal images of root cortical cells of transgenic 8-day old Arabidopsis seedlings. GFP marker lines; false coloured purple: A. plasma membrane, GFP:PM, B. endoplasmic reticulum, GFP:ER, C. tonoplast, GFP:tono. GFP:NtAQP lines: D. NtPIP1;1t, E. NtPIP1;3t, F. NtPIP1;5s, G. NtPIP2;4s, H. NtPIP2;5t, I. NtNIP2;1s, J. NtNIP5;1t, K. NtTIP1;1s and L. NtTIP2;5t. A region of the membrane (indicated by white dashed boxes, $5 \mu \mathrm{m} \times 20 \mu \mathrm{m}$ 
dimension) is magnified in the panel below each confocal image to show surface profiles. Transvacuolar strands are denoted by $\mathrm{V}$. White arrows highlight peak intensity discrepancies present in the NIPs assigned to AQP integration into the ER and PM. Scale bar $5 \mu \mathrm{m}$.

Figure 8. Modelled NtAQP pore features. A. Pore profiles of PIPs (PIP1;1 t, PIP1;3t, PIP1;5s, PIP2;4s, PIP2;5t; blue), TIP1;1s (light purple), TIP2;5t (dark purple), NIP2;1s (dark green) and NIP5;1t (light green). B. A 3D protein model highlighting the Selectivity Filter region (SF, orange residue in 3D Protein model) and NPA region (dark red residues in 3D protein model). C. Amino acid residues forming the selectivity filter and the diameter at its narrowest point, viewed perpendicular to the membrane plane from the extracellular side. D. NPA motifs: NPA 1 and NPA 2 composition of PIPs, TIP1;1s, TIP2;5t, NIP2;1s and NIP5;1t.

Figure 9. Hydrophobicity and flexibility profiles of NtAQP pores. A. 3D Pore model illustrates orientation of the pore profile with the apoplastic entrance (red ball, top), cytosolic entrance (green ball, bottom), residues contacting the pore (yellow balls) and the area inside the AQP pore (blue). Pore profile diameters from ChexVis software (note maximum diameter and pore length scales for each AQP), superimposed with Hydrophobicity (Left hand, Blue to Red indicating low to high hydrophobicity, respectively) and Flexibility (Right hand, Blue to Red indicating low to high flexibility, respectively): B. NtTIPs (NtTIP1;1s and NtTIP25t.) C. NtNIPs (NtNIP2;1s, NtNIP5;1t) and D. NtPIPs (NtPIP1;1t, NtPIP1;3t, NtPIP1;5s, NtPIP2;4s, NtPIP2;5t). Black dots to the left of each pore profile correspond to contact residue interactions for which hydrophobicity and flexibility outputs were 


\section{References}

Abascal F, Irisarri I, Zardoya R (2014) Diversity and evolution of membrane intrinsic proteins. Biochimica et Biophysica Acta (BBA)-General Subjects 1840 (5):1468-1481

Ahmed J, Mercx S, Boutry M, Chaumont F (2020) Evolutionary and predictive functional insights into the aquaporin gene family in the allotetraploid plant Nicotiana tabacum. International journal of molecular sciences 21 (13):4743

Alberti S, Gitler AD, Lindquist S (2007) A suite of Gateway ${ }^{\circledR}$ cloning vectors for high-throughput genetic analysis in Saccharomyces cerevisiae. Yeast 24 (10):913-919

Anderberg HI, Kjellbom P, Johanson U (2012) Annotation of Selaginella moellendorffii major intrinsic proteins and the evolution of the protein family in terrestrial plants. Frontiers in plant science 3:33

Azad AK, Ahmed J, Alum MA, Hasan MM, Ishikawa T, Sawa Y, Katsuhara M (2016) Genome-wide characterization of major intrinsic proteins in four grass plants and their non-aqua transport selectivity profiles with comparative perspective. PLoS One 11 (6):e0157735

Bansal A, Sankararamakrishnan R (2007) Homology modeling of major intrinsic proteins in rice, maize and Arabidopsis: comparative analysis of transmembrane helix association and aromatic/arginine selectivity filters. BMC Structural Biology 7 (1):27

Bi Z, Merl-Pham J, Uehlein N, Zimmer I, Mühlhans S, Aichler M, Walch AK, Kaldenhoff R, Palme K, Schnitzler J-P (2015) RNAi-mediated downregulation of poplar plasma membrane intrinsic proteins (PIPs) changes plasma membrane proteome composition and affects leaf physiology. Journal of proteomics 128:321-332

Bienert GP, Chaumont F (2014) Aquaporin-facilitated transmembrane diffusion of hydrogen peroxide. Biochimica et Biophysica Acta (BBA)-General Subjects 1840 (5):1596-1604

Bienert GP, Desguin B, Chaumont F, Hols P (2013) Channel-mediated lactic acid transport: a novel function for aquaglyceroporins in bacteria. Biochemical Journal 454 (3):559-570

Bienert GP, Møller AL, Kristiansen KA, Schulz A, Møller IM, Schjoerring JK, Jahn TP (2007) Specific aquaporins facilitate the diffusion of hydrogen peroxide across membranes. Journal of Biological Chemistry 282 (2):1183-1192

Bienert MD, Diehn TA, Richet N, Chaumont F, Bienert GP (2018) Heterotetramerization of plant PIP1 and PIP2 aquaporins is an evolutionary ancient feature to guide PIP1 plasma membrane localization and function. Frontiers in plant science 9:382

Byrt CS, Zhao M, Kourghi M, Bose J, Henderson SW, Qiu J, Gilliham M, Schultz C, Schwarz M, Ramesh SA (2017) Non-selective cation channel activity of aquaporin AtPIP2; 1 regulated by Ca2+ and pH. Plant, cell \& environment 40 (6):802-815

Chaumont F, Tyerman S (2017) Plant Aquaporins. Springer,

Chaumont F, Tyerman SD (2014) Aquaporins: Highly regulated channels controlling plant water relations. Plant Physiology 164 (4):1600-1618. doi:10.1104/pp.113.233791

Choi W-G, Roberts DM (2007) Arabidopsis NIP2; 1, a major intrinsic protein transporter of lactic acid induced by anoxic stress. Journal of Biological Chemistry 282 (33):24209-24218

Curtis MD, Grossniklaus U (2003) A gateway cloning vector set for high-throughput functional analysis of genes in planta. Plant physiology 133 (2):462-469

Danielson JÅ, Johanson U (2008) Unexpected complexity of the aquaporin gene family in the moss Physcomitrella patens. BMC Plant Biology 8 (1):45

De Rosa A, Watson-Lazowski A, Evans JR, Groszmann M (2020) Genome-wide identification and characterisation of Aquaporins in Nicotiana tabacum and their relationships with other Solanaceae species. BMC Plant Biology 20 (1):1-29

Deshmukh RK, Sonah H, Bélanger RR (2016) Plant Aquaporins: genome-wide identification, transcriptomics, proteomics, and advanced analytical tools. Frontiers in plant science 7:1896 
Dordas C, Chrispeels MJ, Brown PH (2000) Permeability and channel-mediated transport of boric acid across membrane vesicles isolated from squash roots. Plant physiology 124 (3):13491362

Dynowski M, Schaaf G, Loque D, Moran O, Ludewig U (2008) Plant plasma membrane water channels conduct the signalling molecule H2O2. Biochemical Journal 414 (1):53-61

Fetter K, Van Wilder V, Moshelion M, Chaumont F (2004) Interactions between plasma membrane aquaporins modulate their water channel activity. The Plant Cell 16 (1):215-228

Fitzpatrick KL, Reid RJ (2009) The involvement of aquaglyceroporins in transport of boron in barley roots. Plant, cell \& environment 32 (10):1357-1365

Flexas J, Ribas-Carbó M, Hanson DT, Bota J, Otto B, Cifre J, McDowell N, Medrano H, Kaldenhoff R (2006) Tobacco aquaporin NtAQP1 is involved in mesophyll conductance to CO2in vivo. The Plant Journal 48 (3):427-439

Fox AR, Maistriaux LC, Chaumont F (2017) Toward understanding of the high number of plant aquaporin isoforms and multiple regulation mechanisms. Plant Science 264:179-187

Gomes D, Agasse A, Thiébaud P, Delrot S, Gerós H, Chaumont F (2009) Aquaporins are multifunctional water and solute transporters highly divergent in living organisms. Biochimica et Biophysica Acta (BBA)-Biomembranes 1788 (6):1213-1228

Groszmann M, De Rosa A, Ahmed J, Chaumont F, Evans JR (2021) A consensus on the Aquaporin Gene Family in the Allotetraploid Plant, Nicotiana tabacum. Plant Direct doi:10.1002/PLD3.321

Groszmann M, De Rosa A, Chen W, Qiu J, Byrt CS, Evans JR (in preparation) Characterising the permeability of all 13 Arabidopsis PIP genes using a high throughput yeast approach. . Plant Physiology

Groszmann M, Osborn HL, Evans JR (2017) Carbon dioxide and water transport through plant aquaporins. Plant, Cell \& Environment 40 (6):938-961

Hachez C, Zelazny E, Chaumont F (2006) Modulating the expression of aquaporin genes in planta: a key to understand their physiological functions? Biochimica et Biophysica Acta (BBA)Biomembranes 1758 (8):1142-1156

Halliwell B, Gutteridge JM (2015) Free radicals in biology and medicine. Oxford University Press, USA, Hanaoka H, Uraguchi S, Takano J, Tanaka M, Fujiwara T (2014) OsNIP3; 1, a rice boric acid channel, regulates boron distribution and is essential for growth under boron-deficient conditions. The Plant Journal 78 (5):890-902

Hove RM, Bhave M (2011) Plant aquaporins with non-aqua functions: deciphering the signature sequences. Plant Molecular Biology 75 (4):413-430. doi:10.1007/s11103-011-9737-5

Johanson U, Gustavsson S (2002) A new subfamily of major intrinsic proteins in plants. Molecular Biology and Evolution 19 (4):456-461

Kaldenhoff R, Bertl A, Otto B, Moshelion M, Uehlein N (2007) Characterization of plant aquaporins. In: Methods in enzymology, vol 428. Elsevier, pp 505-531

Kaldenhoff R, Fischer M (2006) Aquaporins in plants. Acta Physiologica 187 (1-2):169-176

Kirscht A, Kaptan SS, Bienert GP, Chaumont F, Nissen P, de Groot BL, Kjellbom P, Gourdon P, Johanson U (2016) Crystal structure of an ammonia-permeable aquaporin. PLoS biology 14 (3):e1002411

Kumar K, Mosa KA, Chhikara S, Musante C, White JC, Dhankher OP (2014) Two rice plasma membrane intrinsic proteins, OsPIP2; 4 and OsPIP2; 7, are involved in transport and providing tolerance to boron toxicity. Planta 239 (1):187-198

Kumar K, Mosa KA, Meselhy AG, Dhankher OP (2018) Molecular insights into the plasma membrane intrinsic proteins roles for abiotic stress and metalloids tolerance and transport in plants. Indian Journal of Plant Physiology 23 (4):721-730

Kumar S, Stecher G, Tamura K (2016) MEGA7: molecular evolutionary genetics analysis version 7.0 for bigger datasets. Molecular biology and evolution 33 (7):1870-1874 
Lee J, Godon C, Lagniel G, Spector D, Garin J, Labarre J, Toledano MB (1999) Yap1 and Skn7 control two specialized oxidative stress response regulons in yeast. Journal of Biological Chemistry 274 (23):16040-16046

Liu L-H, Ludewig U, Gassert B, Frommer WB, von Wirén N (2003) Urea transport by nitrogenregulated tonoplast intrinsic proteins in Arabidopsis. Plant physiology 133 (3):1220-1228

Liu S, Fukumoto T, Gena P, Feng P, Sun Q, Li Q, Matsumoto T, Kaneko T, Zhang H, Zhang Y (2020) Ectopic expression of a rice plasma membrane intrinsic protein (OsPIP1;3) promotes plant growth and water uptake. The Plant Journal 102 (4):779-796

Loqué D, Ludewig U, Yuan L, von Wirén N (2005) Tonoplast intrinsic proteins AtTIP2; 1 and AtTIP2; 3 facilitate NH3 transport into the vacuole. Plant physiology 137 (2):671-680

Luang S, Hrmova M (2017) Structural basis of the permeation function of plant aquaporins. In: Plant Aquaporins. Springer, pp 1-28

Maurel C, Boursiac Y, Luu D-T, Santoni V, Shahzad Z, Verdoucq L (2015) Aquaporins in plants. Physiological reviews 95 (4):1321-1358

Maurel C, Reizer J, Schroeder JI, Chrispeels MJ (1993) The vacuolar membrane protein gamma-TIP creates water specific channels in Xenopus oocytes. The EMBO Journal 12 (6):2241-2247

Maurel C, Verdoucq L, Luu D-T, Santoni V (2008) Plant aquaporins: membrane channels with multiple integrated functions. Annu Rev Plant Biol 59:595-624

Mitani-Ueno N, Yamaji N, Zhao F-J, Ma JF (2011) The aromatic/arginine selectivity filter of NIP aquaporins plays a critical role in substrate selectivity for silicon, boron, and arsenic. Journal of experimental botany 62 (12):4391-4398

Mitani N, Yamaji N, Ma JF (2008) Characterization of substrate specificity of a rice silicon transporter, Lsi1. Pflügers Archiv-European Journal of Physiology 456 (4):679-686

Navarro-RóDenas A, Xu H, Kemppainen M, Pardo AG, Zwiazek JJ (2015) L accaria bicolor aquaporin LbAQP1 is required for $\mathrm{H}$ artig net development in trembling aspen ( $\mathrm{P}$ opulus tremuloides). Plant, cell \& environment 38 (11):2475-2486

Nelson BK, Cai X, Nebenführ A (2007) A multicolored set of in vivo organelle markers for colocalization studies in Arabidopsis and other plants. The Plant Journal 51 (6):1126-1136

Pommerrenig B, Diehn TA, Bienert GP (2015) Metalloido-porins: Essentiality of Nodulin 26-like intrinsic proteins in metalloid transport. Plant Science 238:212-227

Qiu J, McGaughey SA, Groszmann M, Tyerman SD, Byrt CS (2020) Phosphorylation influences water and ion channel function of AtPIP2; 1. Plant, Cell \& Environment 43 (10):2428-2442

Sabir F, Leandro MJ, Martins AP, Loureiro-Dias MC, Moura TF, Soveral G, Prista C (2014) Exploring three PIPs and three TIPs of grapevine for transport of water and atypical substrates through heterologous expression in aqy-null yeast. PloS one 9 (8):e102087

Schindelin J, Arganda-Carreras I, Frise E, Kaynig V, Longair M, Pietzsch T, Preibisch S, Rueden C, Saalfeld S, Schmid B (2012) Fiji: an open-source platform for biological-image analysis. Nature methods 9 (7):676

Soto G, Alleva K, Mazzella MA, Amodeo G, Muschietti JP (2008) AtTIP1; 3 and AtTIP5; 1, the only highly expressed Arabidopsis pollen-specific aquaporins, transport water and urea. FEBS letters 582 (29):4077-4082

Sui H, Han B-G, Lee JK, Walian P, Jap BK (2001) Structural basis of water-specific transport through the AQP1 water channel. Nature 414 (6866):872

Takano J, Wada M, Ludewig U, Schaaf G, Von Wirén N, Fujiwara T (2006) The Arabidopsis major intrinsic protein NIP5; 1 is essential for efficient boron uptake and plant development under boron limitation. The Plant Cell 18 (6):1498-1509

Tanaka M, Wallace IS, Takano J, Roberts DM, Fujiwara T (2008) NIP6; 1 is a boric acid channel for preferential transport of boron to growing shoot tissues in Arabidopsis. The Plant Cell 20 (10):2860-2875 
Tanghe A, Van Dijck P, Dumortier F, Teunissen A, Hohmann S, Thevelein JM (2002) Aquaporin expression correlates with freeze tolerance in baker's yeast, and overexpression improves freeze tolerance in industrial strains. Appl Environ Microbiol 68 (12):5981-5989

Törnroth-Horsefield S, Wang Y, Hedfalk K, Johanson U, Karlsson M, Tajkhorshid E, Neutze R, Kjellbom $P$ (2006) Structural mechanism of plant aquaporin gating. Nature 439 (7077):688

Tyerman SD, McGaughey SA, Qiu J, Yool AJ, Byrt CS (2021) Adaptable and multifunctional ionconducting aquaporins. Annual Review of Plant Biology 72

Uehlein N, Lovisolo C, Siefritz F, Kaldenhoff R (2003) The tobacco aquaporin NtAQP1 is a membrane CO2 pore with physiological functions. Nature 425 (6959):734-737

Wallace IS, Choi W-G, Roberts DM (2006) The structure, function and regulation of the nodulin 26like intrinsic protein family of plant aquaglyceroporins. Biochimica et Biophysica Acta (BBA)Biomembranes 1758 (8):1165-1175

Wallace IS, Roberts DM (2004) Homology modeling of representative subfamilies of Arabidopsis major intrinsic proteins. Classification based on the aromatic/arginine selectivity filter. Plant Physiology 135 (2):1059-1068

Wallace IS, Roberts DM (2005) Distinct transport selectivity of two structural subclasses of the nodulin-like intrinsic protein family of plant aquaglyceroporin channels. Biochemistry 44 (51):16826-16834

Wang S, Yoshinari A, Shimada T, Hara-Nishimura I, Mitani-Ueno N, Ma JF, Naito S, Takano J (2017) Polar localization of the NIP5; 1 boric acid channel is maintained by endocytosis and facilitates boron transport in Arabidopsis roots. The Plant Cell 29 (4):824-842

Wu B, Beitz E (2007) Aquaporins with selectivity for unconventional permeants. Cellular and molecular life sciences 64 (18):2413-2421

Zwiazek JJ, Xu H, Tan X, Navarro-Ródenas A, Morte A (2017) Significance of oxygen transport through aquaporins. Scientific reports 7:40411 


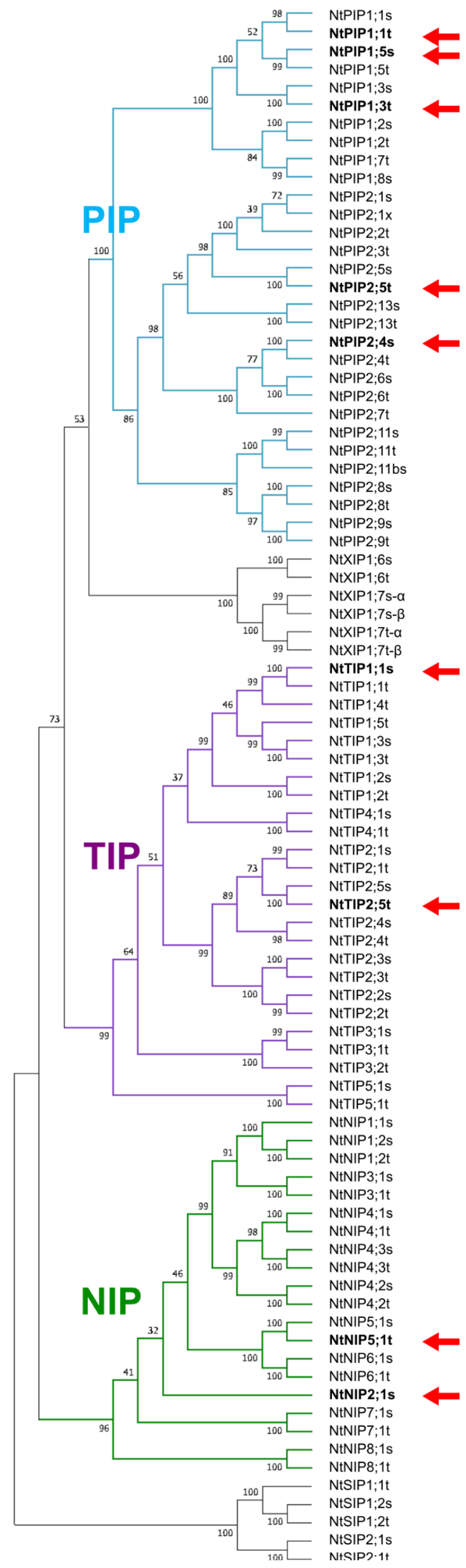

Figure 1. Phylogeny of the NtAQP family, highlighting genes selected for functional characterisation in this study. The phylogenetic tree was generated using the neighbour-joining method from MUSCLE aligned protein sequences. Confidence levels (\%) of branch point generated through bootstrapping analysis $(n=1000)$. Red arrows point to PIP (PIP1;1t, PIP1;3t, PIP1;5s, PIP2;4s, PIP2;5t), TIP (TIP1;1s, TIP2;5t) and NIP (NIP2;1s and NIP5;1t) isoforms functionally characterised in this study. 
Figure 2. Subcellular localisation of GFP tagged aquaporins expressed in yeast. Confocal microscopy images of yeast expressing GFP::NtAQP fusions of A. NtPIP1;1t, B. NtPIP1;3t, C. NtPIP1;5s, D. NtPIP2;4s, E. NtPIP2;5t, F. NtNIP2;1s, G. NtNIP5;1t, H. NtTIP1;1s, I. NtTIP2;5t and J. Free GFP localisation. For each construct we report a Brightfield + GFP overlay image of a yeast cell; a GFP only image; a surface plot profile of GFP signal intensity at the imaged focal plane; and a line scan of signal intensity traversing the cell (indicated by white arrow in GFP only image). Grey shading in GFP signal line scan corresponds to regions which align with the plasma membrane (PM). PM, endoplasmic reticulum (ER) and vacuole (V) are labelled. Scale bar $2 \mu \mathrm{m}$. 

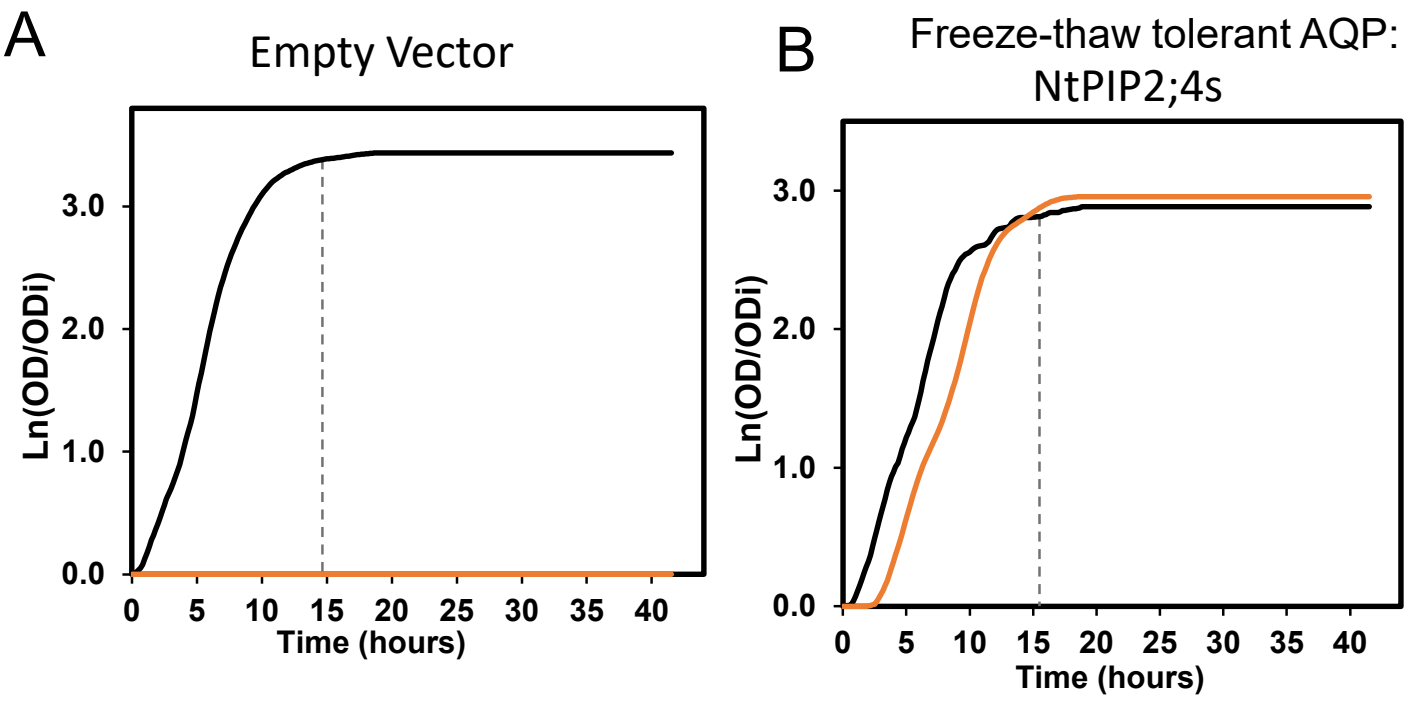

—Untreated $\quad$ Freeze thawed

Untreated

Freeze thawed

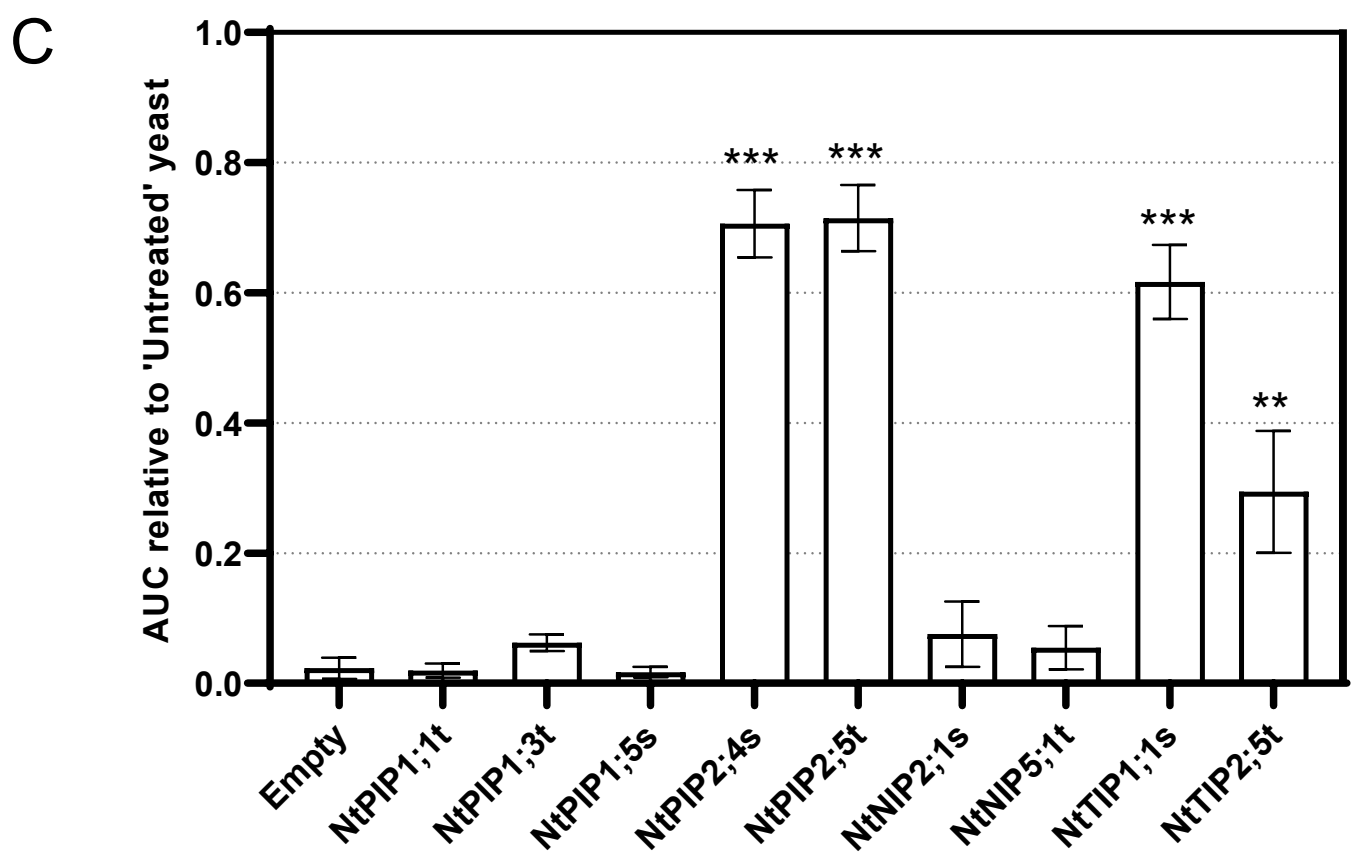

Figure 3. NtAQP water permeability assessed with the 'Freeze-thaw' assay. Yeast growth curves, $\operatorname{Ln}\left(\mathrm{OD} / O D_{\mathrm{i}}\right)$ vs. time, of aqy1 aqy2 yeast expressing $\mathbf{A}$. Empty vector control or $\mathbf{B}$. a freeze-thaw tolerant AQP (NtPIP2;4s), exposed to freeze-thaw treatments. Growth was assessed from the area under the curves (AUC) until the vertical dashed lines. C. Yeast culture growth following the freeze-thaw treatment (AUC relative to untreated yeast control) for aqy1 aqy2 yeast expressing an Empty vector or one of the 9 NtAQPs. Asterisks denote significantly greater growth following the freeze - thaw treatment compared against Empty vector from an ANOVA with Fishers LSD test: “**" $p<0.01$ and "***" $p<0.001, N=6$, Error bars $=S E$. 
A
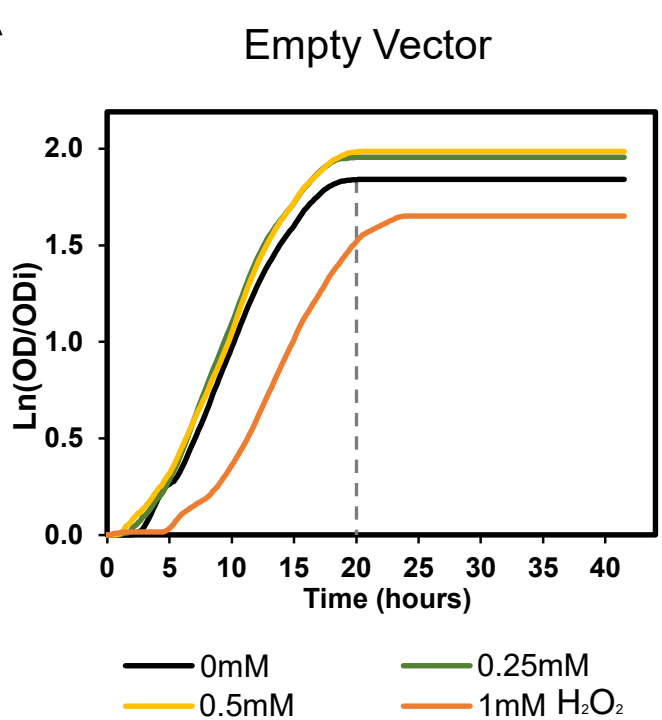

B $\quad \mathrm{H}_{2} \mathrm{O}_{2}$-sensitive AQP: NtPIP2;4s
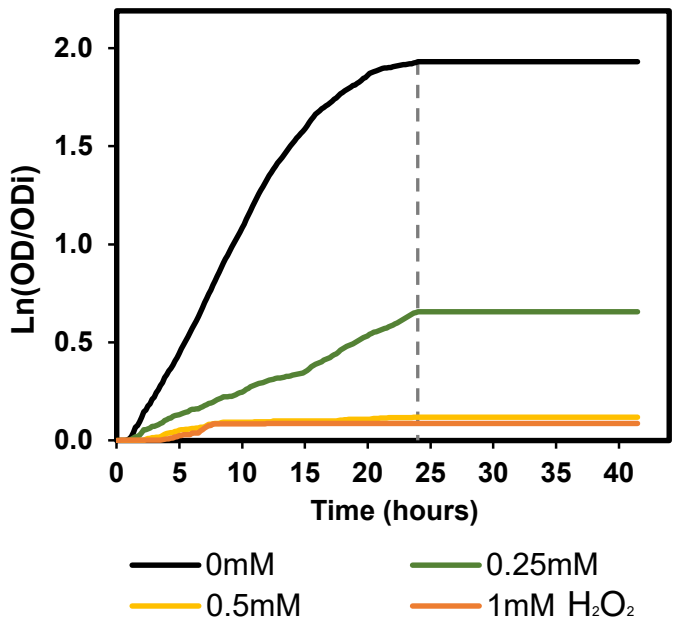

C

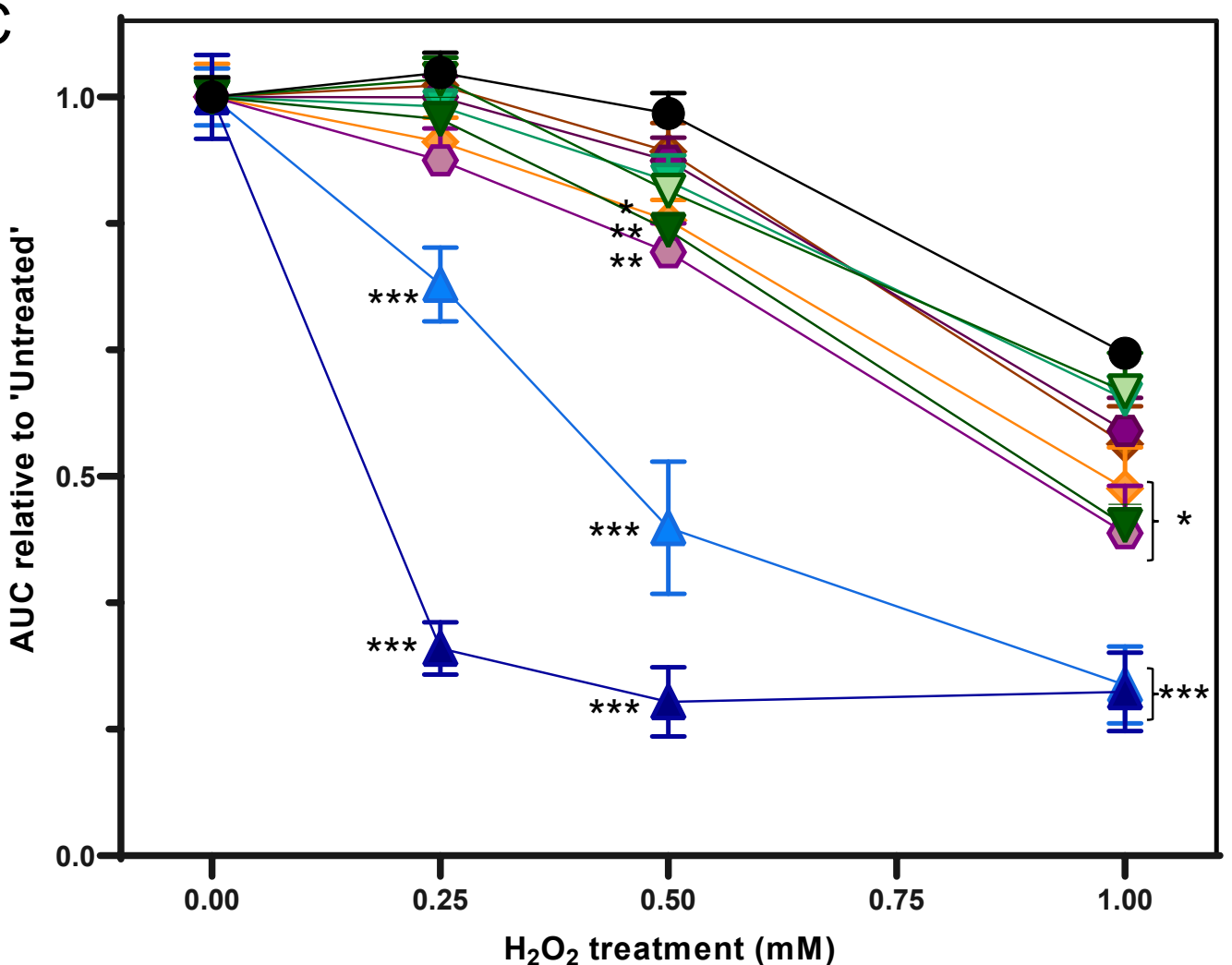

Empty

- NtPIP2;4s

NtTIP1;1s

$\neg$ NtPIP $1 ; 1 \mathrm{t}$

NtPIP2;5t

NtTIP2;5t

$\nabla$ NtPIP1;3t

D. NtNIP2;1s

$\checkmark$ NtPIP1;5s

- NtNIP5;1t

Figure 4. NtAQP $\mathrm{H}_{2} \mathrm{O}_{2}$ permeability assay. Yeast growth curves, $\mathrm{Ln}\left(\mathrm{OD} / O \mathrm{D}_{\mathrm{i}}\right)$ vs. time, of snk7 yeast expressing A. Empty vector control or B. an $\mathrm{H}_{2} \mathrm{O}_{2}$-sensitive AQP (NtPIP2;4s), exposed to $0.25 \mathrm{mM}$, $0.5 \mathrm{mM}$ and $1 \mathrm{mM} \mathrm{H}_{2} \mathrm{O}_{2}$ treatments. Growth was assessed from the area under the curves (AUC) until the vertical dashed lines. C. Yeast culture growth relative to 'Untreated' control (AUC relative to untreated), for skn7 yeast expressing an Empty vector or one the 9 NtAQPs. Asterisks denote Oneway ANOVA with Fishers LSD test results comparing $\mathrm{H}_{2} \mathrm{O}_{2}$-treated yeast growth against Empty vector; "*” $p<0.05$, “**” $p<0.01$ and “***” $p<0.001$. N=6, Error bars=SE. 

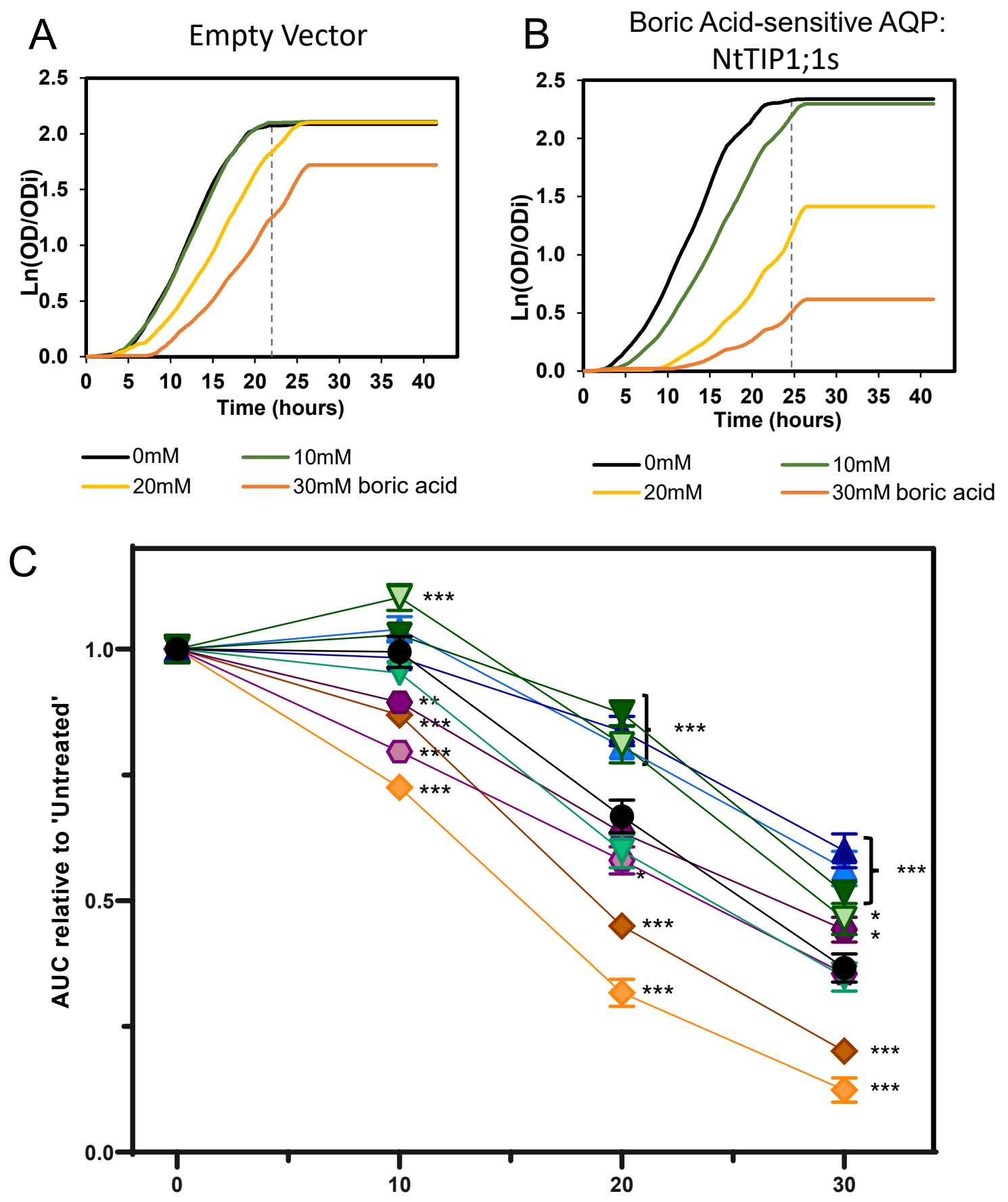

Boric Acid Concentration (mM)
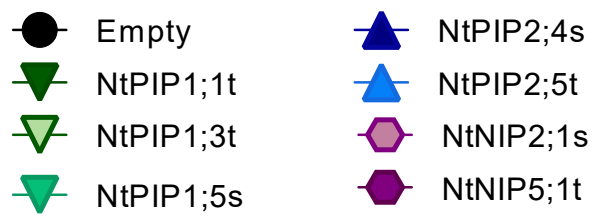

Figure 5. Boric acid permeability assay for yeast expressing NtAQPs. Yeast growth curves, $\operatorname{Ln}\left(O D / O D_{i}\right)$ vs. time, of aqy1 aqy2 yeast expressing $\mathbf{A}$. Empty vector control or $\mathbf{B}$. a boric acid-sensitive AQP (NtTIP1;1s), exposed to $10 \mathrm{mM}, 20 \mathrm{mM}$ and $30 \mathrm{mM}$ boric acid treatments. Growth was assessed from the area under the curves (AUC) until the vertical dashed lines. C. Yeast culture growth relative to untreated control (AUC) of aqy1 aqy2 yeast expressing either an Empty vector or one of the 9 NtAQPs exposed to boric acid. Asterisks denote growth that was significantly different to Empty Vector using a One-Way ANOVA with Fishers LSD test; “*” $p<0.05$, “**” $p<0.01$ and “***” $p<0.001, N=6$, Error bars $=S E$. 
A

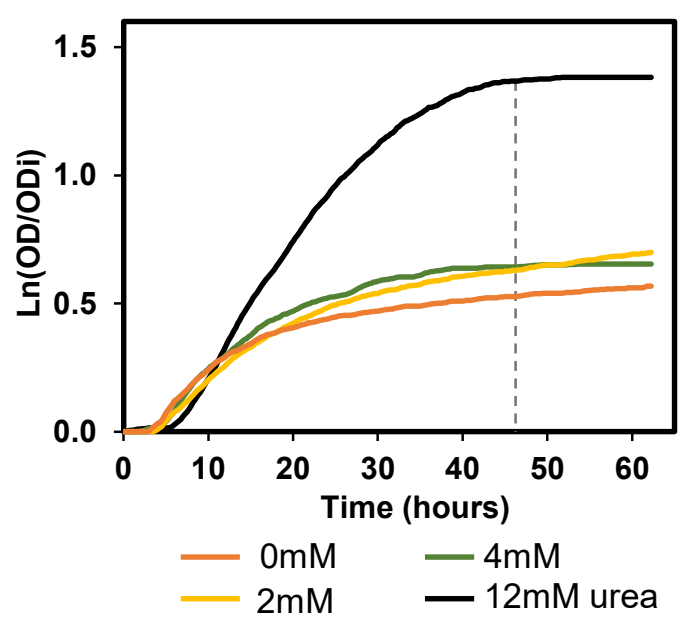

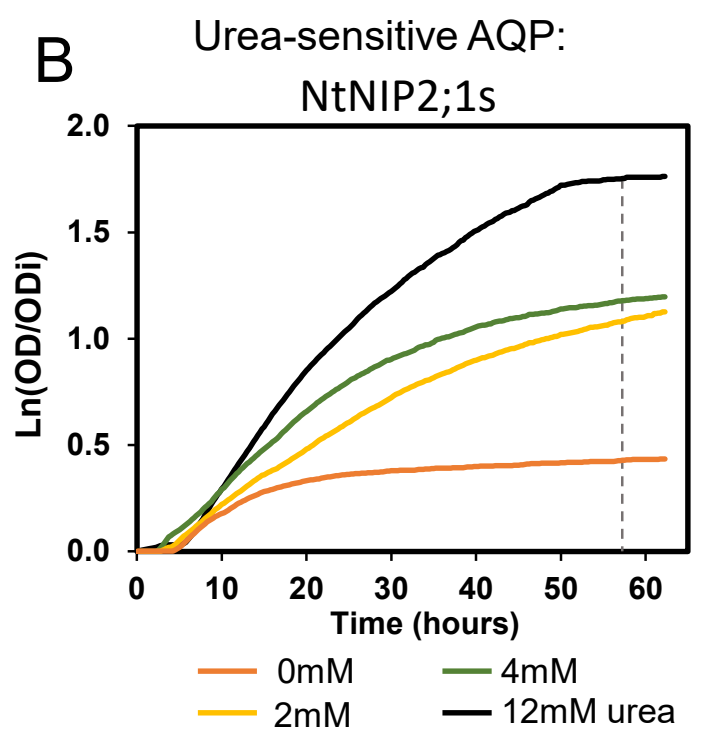

C

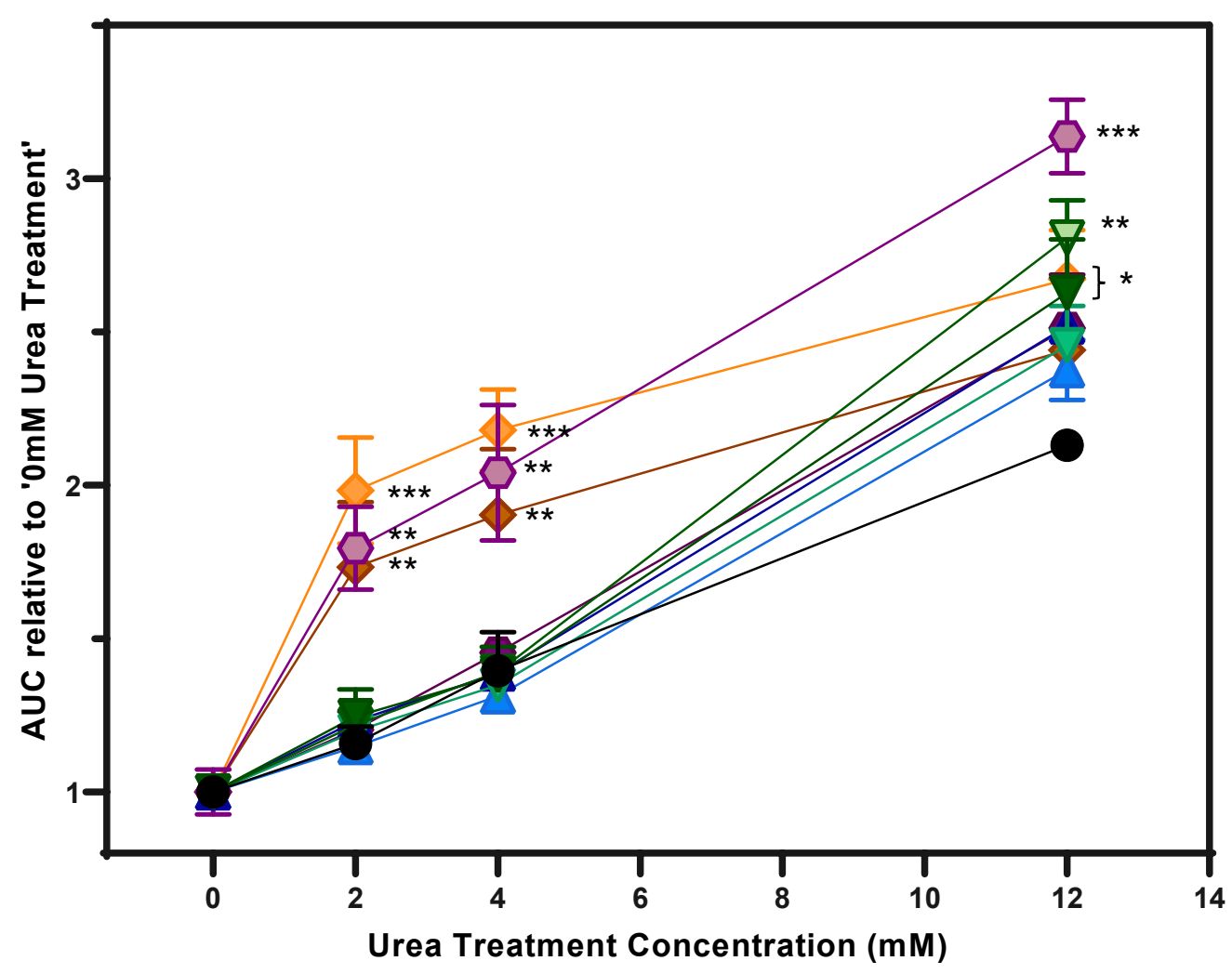

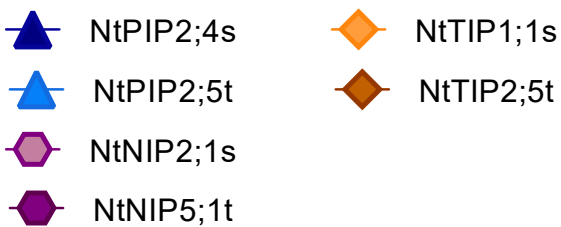

- NtPIP2;4s

NtPIP2;5t

NtNIP5;1t

Figure 6. Urea permeability assays for yeast expressing NtAQPs. Yeast growth curves, $\mathrm{Ln}\left(\mathrm{OD} / O D_{\mathrm{i}}\right)$ vs. time, of ynvwl yeast expressing $\mathbf{A}$. Empty vector control or $\mathbf{B}$. a ureapermeable AQP (NtNIP2;1s), exposed to $0 \mathrm{mM}, 2 \mathrm{mM}, 4 \mathrm{mM}$ or $12 \mathrm{mM}$ urea treatments. Growth was assessed from the area under the curves (AUC) until the vertical dashed lines. C. AUC relative to OmM Urea treatment of ynvwl yeast expressing either an Empty vector or one of the 9 screened NtAQPs. Asterisks denote growth that was significantly greater than Empty vector using a One-way ANOVA with Fishers LSD test, "*" $p<0.05$, "**" $p<0.01$ and “***” $p<0.001$. $N=6$, Error bars=SE. 


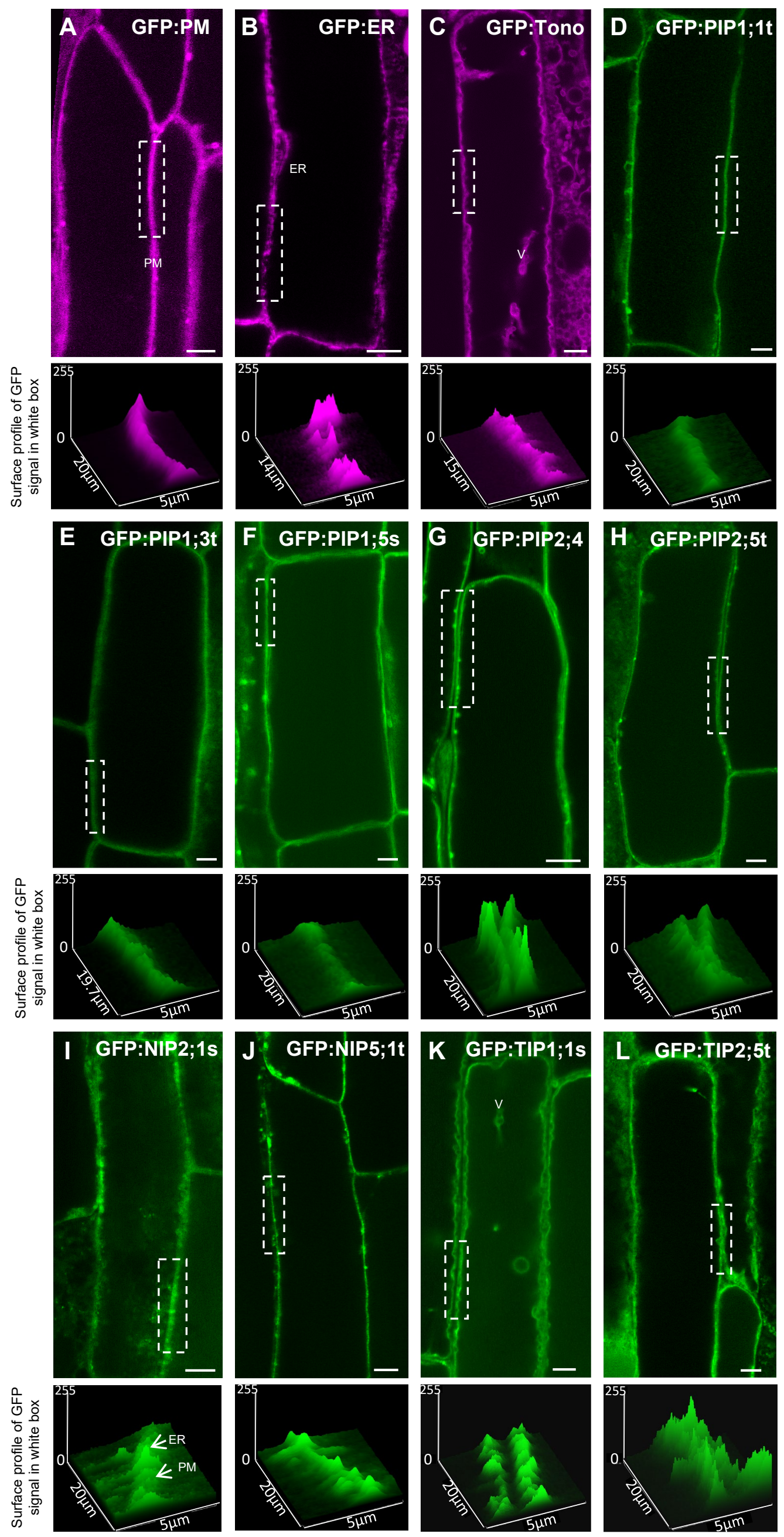


Figure 7. In planta sub-cellular localisation of NtAQPs. Confocal images of root cortical cells of transgenic 8-day old Arabidopsis seedlings. GFP marker lines; false coloured purple: A. plasma membrane, GFP:PM, B. endoplasmic reticulum, GFP:ER, C. tonoplast, GFP:tono. GFP:NtAQP lines: D. NtPIP1;1t, E. NtPIP1;3t, F. NtPIP1;5s, G. NtPIP2;4s, H. NtPIP2;5t, I. NtNIP2;1s, J. NtNIP5;1t, K. NtTIP1;1s and L. NtTIP2;5t. A region of the membrane (indicated by white dashed boxes, $5 \mu \mathrm{m} \times 20 \mu \mathrm{m}$ dimension) is magnified in the panel below each confocal image to show surface profiles. Transvacuolar strands are denoted by V. White arrows highlight peak intensity discrepancies present in the NIPs assigned to AQP integration into the ER and PM. Scale bar $5 \mu \mathrm{m}$. 
A
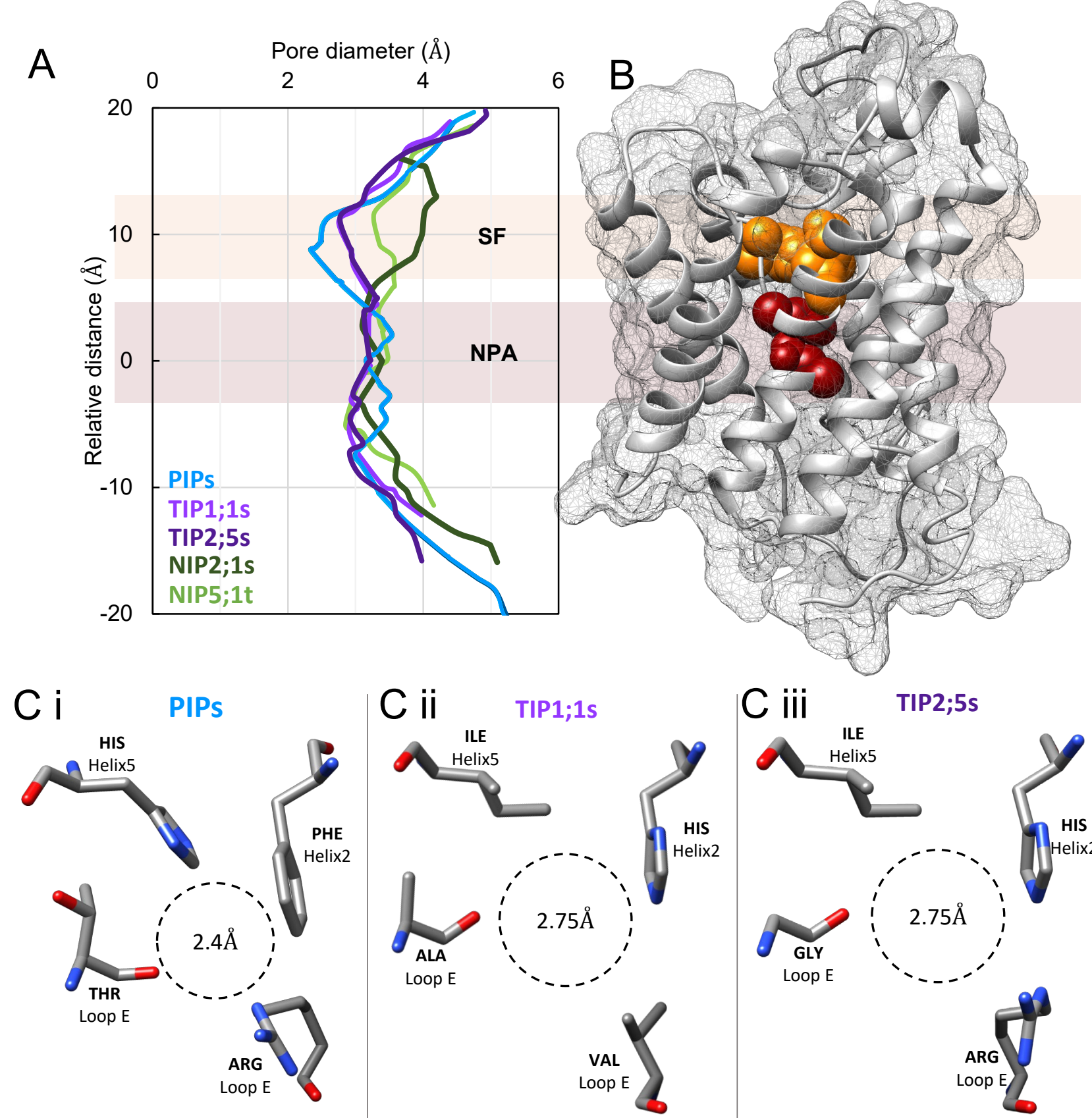

C ii TIP1;1s

C iii TIP2;5s
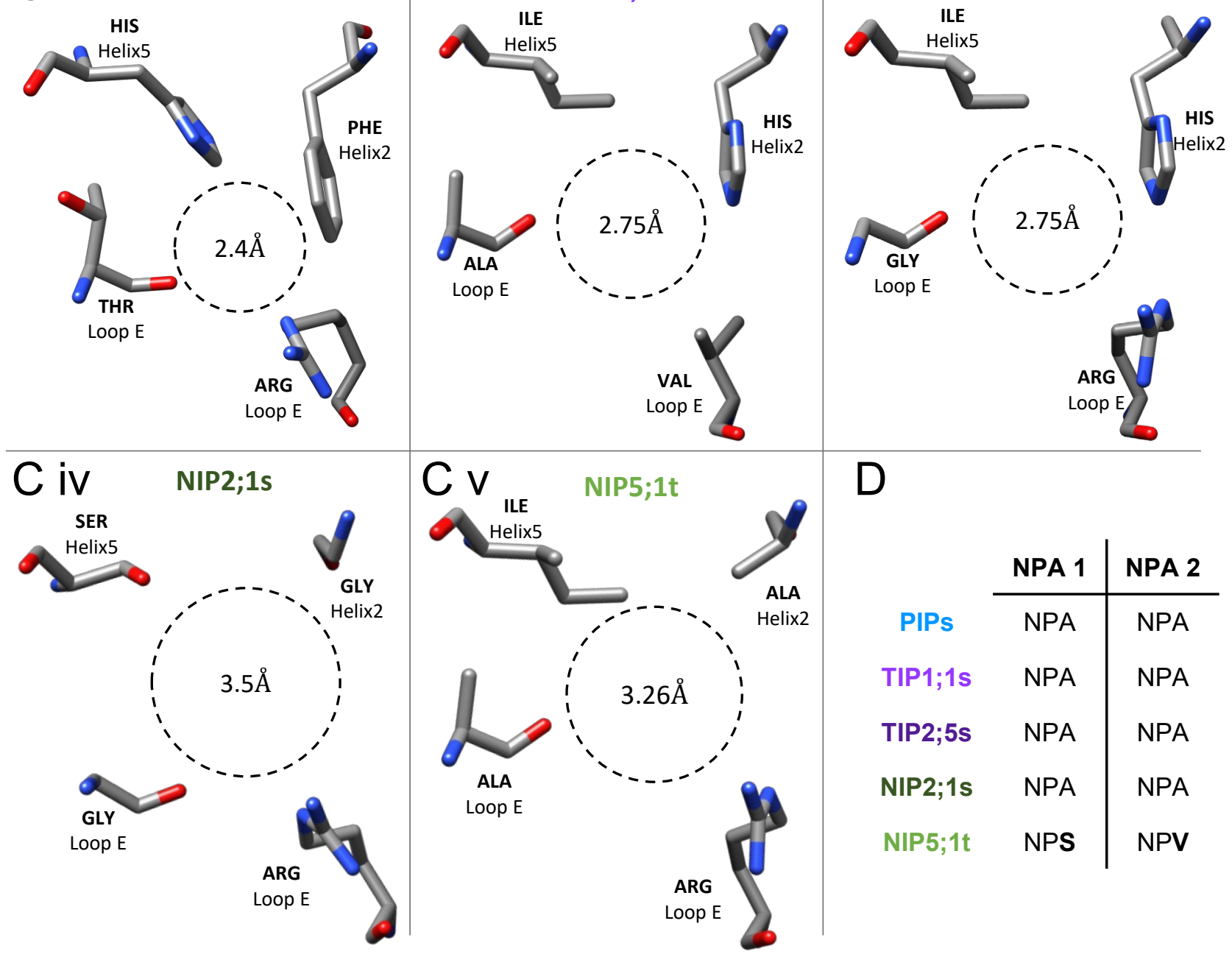

D

\begin{tabular}{cc|c} 
& NPA 1 & NPA 2 \\
\cline { 2 - 3 } PIPs & NPA & NPA \\
TIP1;1s & NPA & NPA \\
TIP2;5s & NPA & NPA \\
NIP2;1s & NPA & NPA \\
NIP5;1t & NPS & NPV
\end{tabular}


Figure 8. Modelled NtAQP pore features. A. Pore profiles of PIPs (PIP1;1 t, PIP1;3t, PIP1;5s, PIP2;4s, PIP2;5t; blue), TIP1;1s (light purple), TIP2;5t (dark purple), NIP2;1s (dark green) and NIP5;1t (light green). B. A 3D protein model highlighting the Selectivity Filter region (SF, orange residue in 3D Protein model) and NPA region (dark red residues in 3D protein model). C. Amino acid residues forming the selectivity filter and the diameter at its narrowest point, viewed perpendicular to the membrane plane from the extracellular side. D. NPA motifs: NPA 1 and NPA 2 composition of PIPs, TIP1;1s, TIP2;5t, NIP2;1s and NIP5;1t. 


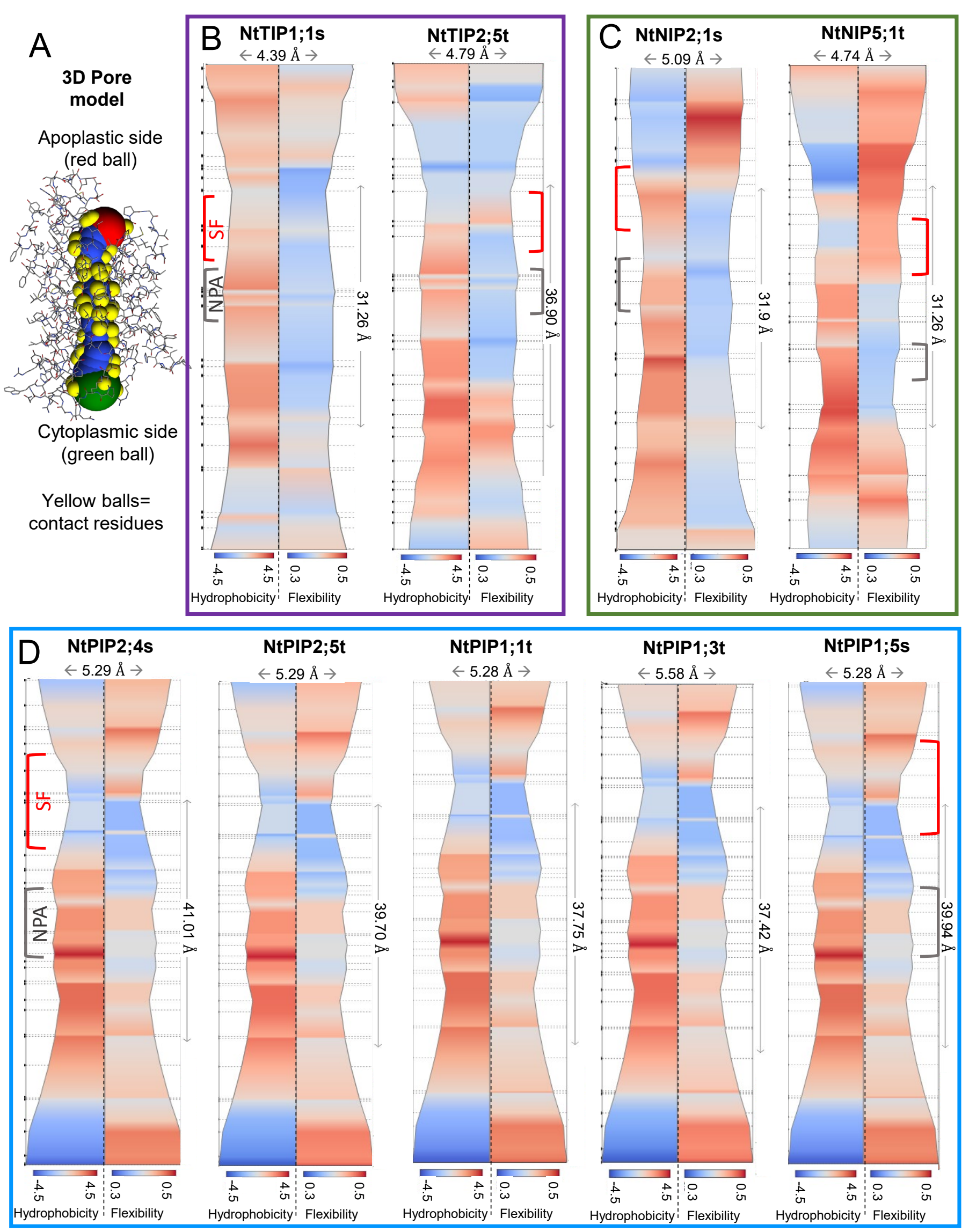


Figure 9. Hydrophobicity and flexibility profiles of NtAQP pores. A. 3D Pore model illustrates orientation of the pore profile with the apoplastic entrance (red ball, top), cytosolic entrance (green ball, bottom), residues contacting the pore (yellow balls) and the area inside the AQP pore (blue). Pore profile diameters from ChexVis software (note maximum diameter and pore length scales for each AQP), superimposed with Hydrophobicity (Left hand, Blue to Red indicating low to high hydrophobicity, respectively) and Flexibility (Right hand, Blue to Red indicating low to high flexibility, respectively): B. NtTIPs (NtTIP1;1s and NtTIP25t.) C. NtNIPs (NtNIP2;1s, NtNIP5;1t) and D. NtPIPs (NtPIP1;1t, NtPIP1;3t, NtPIP1;5s, NtPIP2;4s, NtPIP2;5t). Black dots to the left of each pore profile correspond to contact residue interactions for which hydrophobicity and flexibility outputs were generated. Red brackets indicate Selectivity Filter (SF) region and grey brackets indicate NPA region. 